\title{
ON LINEAR TRANSFORMATIONS
}

\author{
BY
}

\author{
R. S. PHILLIPS
}

The purpose of this paper is to give a characterization of linear and com. pletely continuous transformations both on the common Banach spaces to an arbitrary Banach space and vice versa. There is an abundant literature on this subject. Among the earliest papers, the now famous paper of Radon [24] should be mentioned. Here linear transformations on $L^{p}$ to $L^{q}(1<p, q<\infty)$ are characterized in a manner suggestive of the methods used in the present paper. The works of Gelfand [12], Dunford [6], Kantorovitch and Vulich [17], and Dunford and Pettis [9] contain much material on this subject supplementary to that treated here. In the interest of completeness we have restated a few of the results obtained by Gelfand [12], and Gowurin [13].

The principal tools used in our characterizations are certain abstractly valued function spaces. One such space is the class of all additive set functions $x(\tau)$ on all Lebesgue measurable subsets $\tau$ of $(0,1)$ to a Banach space $X$ where for all linear functionals $\bar{x}$ on $X$ and for all subdivisions $\pi=\left(\tau_{1}, \tau_{2}, \cdots, \tau_{n}, \cdots\right)$ of $(0,1)$ into disjoint sets,

$$
\underset{\pi}{\text { L.U.B. }}\left[\sum_{\pi} \frac{\left|\bar{x}\left[x\left(\tau_{i}\right)\right]\right|^{q}}{\left|\tau_{i}\right|^{q-1}}\right]<\infty \text {. }
$$

If $\phi(t) \varepsilon L^{p}(1 / p+1 / q=1)$, we define an integral $\int \phi d x$ to be the generalized $\pi$-limit of the unconditionally convergent sums $\sum_{x} \phi\left(t_{i}\right) x\left(\tau_{i}\right)$ where $t_{i} \varepsilon \tau_{i}$. The function $U(\phi)=\int \phi d x$ so defined on $L^{p}$ is a characterization of the general linear transformation on $L^{p}$ to $X$.

The first section is a study of the abstractly valued function spaces which will be used to characterize the transformations. Section 2 is devoted to a discussion of three different types of integrals needed in these characterizations. In $\S 3$ a necessary and sufficient condition for a subset of a Banach space $Y$ to be conditionally compact is given in terms of an arbitrary determining manifold $\Gamma$ in the conjugate space $\bar{Y}$. As a consequence, if a transformation $U$ is additive and homogeneous on $X$ to $Y$ and its adjoint is completely continuous on $\Gamma$ to $\bar{X}$, then $U$ is completely continuous on $X$ to $Y$. The section also contains a characterization of conditionally compact sets in a Banach space by means of a generalized base. This is applied to the spaces $L^{p}(1 \leqq p \leqq \infty)$ in $\$ \S 5$ and 6 . Section 4 contains the principal results of this paper, namely, a characterization for the classes of transformations considered. In $\$ 5$ we

Presented to the Society, December 29, 1939; received by the editors March 9, 1940. This paper was received by the editors of the Annals of Mathematics November 18, 1939, accepted by them, and later transferred to these Transactions. 
obtain representations by means of a kernel of the general completely continuous transformation and weakly completely continuous separable transformation on $L$ to an arbitrary Banach space. By means of this result and a theorem due to Dunford and Pettis [9], we show that $U^{2}$ is completely continuous whenever $U$ on $L$ to $L$ is weakly completely continuous. As a further application of this work, we prove in $\$ 6$ that completely continuous transformations on the spaces $L^{p}, l^{p}, C, c_{0}, M_{T}(1 \leqq p \leqq \infty)$ to an arbitrary Banach space are approximable in the norm by degenerate transformations. A final section is devoted to the extension of linear transformations.

We will consider an abstract class $T$ of elements $t$ possessing a sigma family $\tau$ of subsets $\tau$ of $T . \alpha(\tau)$ will be a single-valued, non-negative, completely additive measure function on $\mathcal{T}$, which need not be finite valued. It will be convenient to designate by $|\tau|$ the value $\alpha(\tau) . X$ will denote a Banach space of elements $x$ and $\bar{X}$ its conjugate space of elements $\bar{x}$ [1, chap. 5]. We define with Dunford $[7$, p. 316] a determining manifold $\Gamma$ in $\bar{X}$ to be a closed linear subset of $\bar{X}$ such that( $\left.{ }^{1}\right)$ L.U.B. $[|\bar{x}(x)| \mid x \varepsilon X, \bar{x} \varepsilon \Gamma,\|\bar{x}\| \leqq 1]=\|x\|$. $M_{T}$ will be the Banach space of bounded functions $a(t)$ on an abstract class $T=[t]$ to real numbers having the norm $\|a\|=$ L.U.B. $[|a(t)| \mid t \varepsilon T] . \pi$ will have three different meanings: type $1, \pi$ will be a finite or denumerable set of disjoint sets $\tau$ of $\mathcal{T}$ such that $0<|\tau|<\infty . \pi_{1} \geqq \pi_{2}$ will mean that $\sum \tau^{1} \supset \sum \tau^{2}$, and that every set $\tau^{1} \varepsilon \pi_{1}$ is either a subset of some $\tau^{2} \varepsilon \pi_{2}$ or $\tau^{1}$ is disjoint from every $\tau^{2} \varepsilon \pi_{2}$; type $2, \pi$ will be a subdivision of $T$ into a finite number of disjoint sets $\tau \boldsymbol{\varepsilon} \mathcal{T}$ ( $\mathcal{T}$ need not possess a measure function). $\pi_{1} \geqq \pi_{2}$ will mean that each $\tau^{1} \varepsilon \pi_{1}$ is a subset of some $\tau^{2} \varepsilon \pi_{2}$; and type $3, \pi$ will be a subdivision of the interval $(0,1)$ into a finite number of intervals the maximum of whose lengths is $|\pi| . \pi_{1} \geqq \pi_{2}$ will mean that $\left|\pi_{1}\right| \leqq\left|\pi_{2}\right|$. In each case the relation $\geqq$ on the class $[\pi]$ is transitive and compositive. The general limit of E. H. MooreH. L. Smith $[20$, p. 103] can therefore be defined on each of these ranges. $\operatorname{Lim}_{\pi}$ will designate this limit.

1. Abstractly valued function spaces. We will be interested in the following classes of functions:

$$
\begin{aligned}
& V^{1}(X, \Gamma) \equiv\left[x(\tau)\left|\underset{\pi}{\text { L.U.B. }} \sum_{\boldsymbol{x}}\right| \bar{x}\left[x\left(\tau_{i}\right)\right] \mid<\infty, \quad \bar{x} \varepsilon \Gamma\right], \\
& V^{q}(X, \Gamma) \equiv\left[x(\tau) \mid \text { L.U.B. } \sum_{\pi} \frac{\left|\bar{x}\left[x\left(\tau_{i}\right)\right]\right|^{q}}{\left|\tau_{i}\right|^{q-1}}<\infty, \bar{x} \varepsilon \Gamma\right], \quad 1<q<\infty, \\
& V^{\infty}(X) \equiv[x(\tau)|\|x(\tau)\| \leqq M \cdot| \tau|, \tau \varepsilon \mathcal{T},| \tau \mid<\infty], \\
& v^{q}(X, \Gamma) \equiv\left[\left.x_{n}\left|\sum_{n}\right| \bar{x}\left(x_{n}\right)\right|^{q}<\infty, \bar{x} \varepsilon \Gamma\right], \quad 1 \leqq q<\infty \\
& v^{\infty}(X) \equiv\left[x_{n} \mid \underset{n}{\text { L.U.B. }}\left\|x_{n}\right\|<\infty\right] \text {. }
\end{aligned}
$$

(1) The class of elements $s$ satisfying the property $P$ will be designated by $[s \mid P]$. 
For $q \neq 1(q=1) \pi$ is always to be understood as being of type 1 (type 2). For $q=1, \mathcal{T}$ may be a finitely additive Jordan field. It will be convenient to denote an element of one of these function classes by $\dot{x}$.

If $T$ is the set of positive integers, $\mathcal{T}$ the family of all subsets of $T$, and if $|\tau|$ is equal to the number of integers in $\tau$, then $V^{q}(X, \Gamma)(1<q<\infty)$ and $V^{\infty}(X)$ are identical with $v^{q}(X, \Gamma)$ and $v^{\infty}(X)$ respectively. Theorems analogous to Theorems $1.1,1.2$, and 1.5 have been proved for $v^{1}(X, \Gamma)$ by Gelfand [12].

1.1. Theorem. If $\dot{x} \varepsilon V^{q}(X, \Gamma), 1 \leqq q<\infty$, then there exists an $M$ such that $\left({ }^{2}\right)$

$$
\underset{\pi}{\text { L.U.B. }}\left[\sum_{\pi} \frac{\left|\bar{x}\left[x\left(\tau_{i}\right)\right]\right|^{q}}{\left|\tau_{i}\right|^{q-1}}\right]^{1 / q} \leqq M \cdot\|\bar{x}\|
$$

for all $\bar{x} \varepsilon \Gamma$.

Define

$$
p(\bar{x})=\underset{\pi}{\operatorname{L} . U . B .}\left[\sum_{\pi} \frac{\left|\bar{x}\left[x\left(\tau_{i}\right)\right]\right|^{q}}{\left|\tau_{i}\right|^{q-1}}\right]^{1 / q}
$$

on $\Gamma$. It is easy to show that $p(\bar{x}) \geqq 0, p\left(\bar{x}_{1}+\bar{x}_{2}\right) \leqq p\left(\bar{x}_{1}\right)+p\left(\bar{x}_{2}\right)$, and that $\bar{x}_{n} \rightarrow \bar{x}$ implies $\lim \inf _{n \rightarrow \infty} p\left(\bar{x}_{n}\right) \geqq p(\bar{x})$. The theorem now follows from a lemma due to Gelfand [12, p. 240].

We define a norm for the several spaces as follows:

$$
\begin{aligned}
& \|\dot{x}\|=\underset{\|\bar{x}\|=1}{\text { L.U.B. }}\left\{\underset{\pi}{\text { L.U.B. }}\left[\sum_{\pi} \frac{\left|\bar{x}\left[x\left(\tau_{i}\right)\right]\right|^{q}}{\left|\tau_{i}\right|^{q-1}}\right]^{1 / q} \mid \bar{x} \varepsilon \Gamma\right\}, \\
& \dot{x} \varepsilon V^{q}(X, \Gamma), 1 \leqq q<\infty, \\
& \|\dot{x}\|=\text { L.U.B. }\left[\frac{\|x(\tau)\|}{|\tau|}|\tau \varepsilon \mathcal{G},| \tau \mid<\infty\right] \text {, } \\
& \dot{x} \varepsilon V^{\infty}(X) \text {, } \\
& \|\dot{x}\|=\text { L.U.B. }\left[\sum_{n}\left|\bar{x}\left(x_{n}\right)\right|, \bar{x} \varepsilon \Gamma\right] \text {, } \\
& \dot{x} \varepsilon v^{1}(X, \Gamma) \text {. }
\end{aligned}
$$

1.2. Theorem. $V^{q}(X, \Gamma), 1 \leqq q \leqq \infty$, is a Banach space.

It is clear that the spaces are linear normed spaces. Only the proof of completeness remains. Suppose $\left\{\dot{x}_{n}\right\}$ is a Cauchy sequence in $V^{q}(X, \Gamma), 1 \leqq q<\infty$. Then for every $\tau \varepsilon \mathcal{\tau}$,

$$
\lim _{m, n \rightarrow \infty} \frac{\left|\bar{x}\left[x_{n}(\tau)-x_{m}(\tau)\right]\right|^{q}}{|\tau|^{q-1}}=0
$$

uniformly in the unit sphere of $\Gamma$. Hence $\lim _{m, n \rightarrow \infty}\left\|x_{m}(\tau)-x_{n}(\tau)\right\|=0$ and there exists an additive set function $x(\tau)=\lim _{n \rightarrow \infty} x_{n}(\tau)$. Further, if we are given $\bar{x}$ in

(2) For $q=1$, we define $|\tau|^{q^{-1}}$ to be identically one. 
the unit sphere of $\Gamma, \pi$, and $N$, then

$$
\left[\sum_{1}^{N} \frac{\left|\bar{x}\left[x\left(\tau_{i}\right)\right]\right|^{q}}{\left|\tau_{i}\right|^{q-1}}\right]^{1 / q} \leqq \lim _{n}\left\|\dot{x}_{n}\right\|
$$

so that $\dot{x} \varepsilon V^{q}(X, \Gamma)$. Finally for an arbitrary $e>0$ there exists $N_{e}$ such that if $m, n \geqq N_{e}$ then $\left\|\dot{x}_{m}-\dot{x}_{n}\right\| \leqq e$. Therefore if $n>N_{e}$

$$
\left\|\dot{x}-\dot{x}_{n}\right\|=\underset{\|\tilde{x}\|=1, \pi, N}{\text { L.U.B. }}\left\{\lim _{m}\left[\sum_{1}^{N} \frac{\left|\bar{x}\left[x_{n}\left(\tau_{i}\right)-x_{m}\left(\tau_{i}\right)\right]\right|^{q}}{\left|\tau_{i}\right|^{q-1}}\right]^{1 / q}\right\} \leqq e .
$$

Completeness for $V^{\infty}(X)$ can be demonstrated in a similar fashion.

If $X$ is the space of real numbers $R$, then $\Gamma$ must be identical with $\bar{X}=R$. For $T=\sum \tau_{1}\left(\left|\tau_{i}\right|<\infty\right)$ it is well known that $V^{q}(R)(1 \leqq q \leqq \infty)$ is equivalent $\left[1\right.$, p. 180] to the space $L^{q}(\alpha)$ of measurable functions $\psi(t)$ for which $\int_{T}|\psi(t)|^{q} d \alpha<\infty(1 \leqq q<\infty)$ and ess. L.U.B. $[|\psi(t)| \mid t \varepsilon T]<\infty(q=\infty)$. The equivalence is defined by the transformation $U(\psi)=x(\tau)=\int_{\tau} \psi(t) d \alpha$ for $|\tau|<\infty$.

A sum $\sum x_{n}$ will be said to be unconditionally convergent if $\sum x_{n}$ summed over any subsequence of the integers converges. $x(\tau)$ will be called completely additive if for any sequence of disjoint measurable sets $\left\{\tau_{n}\right\}, \sum x\left(\tau_{n}\right)=x\left(\sum \tau_{n}\right)$ where the sum is unconditionally convergent.

1.3. TheOREM. If $\dot{x} \varepsilon V^{q}(X, \Gamma)(1<q \leqq \infty)$ and if $\tau_{0}$ is of finite measure, then $x(\tau)$ is an absolutely continuous and completely additive set function on measurable subsets of $\tau_{0}$.

If $\dot{x} \varepsilon V^{q}(X, \Gamma)$, then

$$
\left[\frac{|\bar{x}[x(\tau)]|^{q}}{|\tau|^{q-1}}\right]^{1 / q} \leqq\|\dot{x}\| \cdot\|\bar{x}\|
$$

for all $\bar{x} \varepsilon \Gamma$ and all $\tau(0<|\tau|<\infty)$. Hence $\|x(\tau)\| \leqq\|\dot{x}\| \cdot|\tau|^{1-1 / q}$ which implies absolute continuity. Let us now consider $x(\tau)$ and $V^{q}(R)$ on subsets of a set $\tau_{0}$ of finite measure. Since $\bar{x}[x(\tau)] \varepsilon V^{q}(R)$ it follows from the above that there exists $\psi(t) \varepsilon L^{q}(\alpha)$ for which $\bar{x}[x(\tau)]=\int_{\tau} \psi(t) d \alpha$. Given a sequence $\left\{\tau_{n}\right\}$ of disjoint measurable sets, then

$$
\bar{x}\left[x\left(\sum \tau_{n}\right)\right]=\int_{\Sigma \tau_{n}} \psi d \alpha \equiv \sum \int_{\tau_{n}} \psi d \alpha=\sum \bar{x}\left[x\left(\tau_{n}\right)\right] .
$$

By a theorem due to Dunford [7, p. 326, Theorem 32], $x\left(\sum \tau_{n}\right)=\sum x\left(\tau_{n}\right)$ which is unconditionally convergent.

It is clear that the transformation $U(\bar{x})=\bar{x}[x(\tau)]$ on $\Gamma$ to $V^{q}(R)$ $(1 \leqq q \leqq \infty)$ is linear and that $\|U\|=\|\dot{x}\|$. We define $V^{q} c(X, \Gamma)$ to be the subspace of $V^{q}(X, \Gamma)$ for which this transformation is completely continuous. Because the class of completely continuous transformations on $X$ to $Y$ is a 
closed linear subspace of the space of linear transformations, it follows that the same is true of $V^{q} c(X, \Gamma)$ in $V^{q}(X, \Gamma)$. We define $v^{q}(X, \Gamma)(1 \leqq q \leqq \infty)$ in a similar fashion.

1.4. Theorem. A necessary and sufficient condition that $\dot{x}$ belong to $V^{\infty}{ }_{c}(X)$ is that $\dot{x}$ belong to $V^{\infty}(X)$ and that the set $[x(\tau) /|\tau| \mid \tau \varepsilon \mathcal{G}]$ be conditionally compact.

This is an immediate consequence of Theorem 3.1.

1.5. Theorem. A necessary and sufficient condition that $\dot{x}$ belong to $V^{1}{ }_{c}(X, \Gamma)$ is that $\dot{x}$ belong to $V^{1}(X, \Gamma)$ and that the set $[x(\tau) \mid \tau \varepsilon \mathcal{\tau}]$ be conditionally compact.

This again follows from Theorem 3.1.

1.6. Theorem. If $T=(0,1)$ and $\alpha$ is the Lebesgue measure function, then for $1<q<\infty$ the following are equivalent statements:

(1) $\dot{x} \varepsilon V^{q} c(X, \Gamma)$.

(2) $\dot{x} \varepsilon V^{q}(X, \Gamma)$ and

$$
\left.\left.\lim _{h \rightarrow 0} \int_{0}^{1} \mid \frac{d \bar{x}\left[x\left(I_{0}^{t}\right)\right]}{d t}\right]_{t+h}-\frac{d \bar{x}\left[x\left(I_{0}^{t}\right)\right]}{d t}\right]_{t}^{q} d t=0
$$

uniformly for all $\bar{x}$ in the unit sphere of $\Gamma\left(I_{0}^{t}=(0, t)\right)$.

This follows from the above remarks on the equivalence between $V^{q}(R)$ and $L^{q}$ and a theorem due to M. Riesz on compact sets in $L^{q}$ [25].

1.7. Theorem. A necessary and sufficient condition that $\dot{x}$ belong to $v_{c}{ }_{c}(X, \Gamma)$ for $1 \leqq q<\infty$ is that $\dot{x}$ belong to $v^{q}(X, \Gamma)$ and that $\lim _{n \rightarrow \infty} \sum_{n}^{\infty}\left|\bar{x}\left(x_{i}\right)\right|^{q}=0$ uniformly in the unit sphere of $\Gamma$.

This follows from a well known theorem on compact sets in $l^{q}$, which for $q=2$ is due to Fréchet $[11$, p. 19].

If $\dot{x} \varepsilon v^{1}(X, \Gamma)$, then $\left\|\sum_{\nu} x_{n}\right\| \leqq\|\dot{x}\| \leqq 2 \cdot$ L.U.B. $\left\|\sum_{\nu} x_{n}\right\|$ where $\nu$ runs through all finite sets of integers. If $\dot{x} \varepsilon v^{1} c(X, \Gamma)$, we have as a corollary to Theorem 1.7 that $\sum x_{n}$ is unconditionally convergent. Dunford has shown that if $\sum x_{n}$ is unconditionally convergent, then $\dot{x} \varepsilon v^{1}{ }_{c}(X, \Gamma)[7$, p. 326, Theorem 32].

1.8. TheOREM. If $\bar{X}$ is separable and if $\dot{\bar{x}} \varepsilon v^{1}(\bar{X}, \Gamma)$, then $\dot{\bar{x}} \varepsilon v^{1}(\bar{X}, \overline{\bar{X}})$.

By hypothesis $\sum\left|\bar{x}_{n}(x)\right| \leqq\|\dot{x}\| \cdot\|x\|$ for every $x \varepsilon X$. Hence for every denumerable set of integers $\sigma,\left|\sum_{\sigma} \bar{x}_{n}(x)\right| \leqq\|\dot{x}\| \cdot\|x\|$ and is therefore a linear functional $\bar{x}_{\sigma}$ on $X$. Since $X$ is a determining manifold in $\bar{X}$, it follows by the above mentioned Dunford theorem that $\sum x_{n}$ is unconditionally convergent. In other words $\dot{\bar{x}} \varepsilon v^{1}(\bar{X}, \bar{X})$. 
1.9. Corollary. If $X$ is separable and there exists an $\dot{x} \varepsilon v^{1}(X, \bar{X})$ which is not an element of $v_{c}^{1}(X, \bar{X})$, then $X$ and any separable space containing $X$ as a subspace are not conjugate spaces.

This permits another demonstration of the fact that $c$ and hence $C$ which contains $c$ is not a conjugate space. Let $x_{n}$ be the $n$th unit vector in $c$. Any $\bar{x}=\left\{a_{n}\right\} \varepsilon l\left[1\right.$, p. 67]. $\sum\left|\bar{x}\left(x_{n}\right)\right|=\sum\left|a_{n}\right|<\infty$ implies that $\dot{x} \varepsilon v^{1}(c, l)$. However $\sum x_{n}$ is obviously not unconditionally convergent.

2. Integrals. It is convenient to divide the discussion of this section into three parts: (1) an integral involving functions $\phi(t) \varepsilon L^{p}(\alpha)$ and $\dot{x} \varepsilon V^{q}(X, \Gamma)$ where $1 / p+1 / q=1$ and $1<q \leqq \infty ;(2)$ an integral involving functions $\phi(t)$ either bounded or $\alpha$-measurable and essentially bounded and $\dot{x} \varepsilon V^{1}(X, \Gamma)$; and (3) an integral involving functions $\phi(t) \varepsilon C$ and functions $x(t)$ to be defined.

2.1. Definition. For functions $\phi(t) \varepsilon L^{p}(\alpha)$ and $\dot{x} \varepsilon V^{q}(X, \Gamma), 1<q \leqq \infty$, we define

$$
\int \phi d x=\lim _{\pi} \sum_{\pi} \phi\left(t_{i}\right) x\left(\tau_{i}\right)
$$

whenever for some $\pi_{0}$ and all $\pi \geqq \pi_{0}, \sum_{\pi} \phi\left(t_{i}\right) x\left(\tau_{i}\right)$ is unconditionally convergent for each $t_{i} \varepsilon \tau_{i}$ and the limit exists.

The multiple valued function $x(\pi)$ on a transitive and compositive class $[\pi]$ will be said to be a fundamental $\pi$-sequence if for an arbitrary $e>0$ there exists a $\pi_{e}$ such that for $\pi_{1}, \pi_{2} \geqq \pi_{e},\left\|x\left(\pi_{1}\right)-x\left(\pi_{2}\right)\right\| \leqq e\left(^{3}\right)$.

2.2. Lemma. If $[x(\pi)]$ is a fundamental $\pi$-sequence and $U$ a linear transformation on $X$ to $Y$, then there exists an $x \varepsilon X$ such that $x=\lim _{\pi} x(\pi)$ and $U(x)=\lim _{\pi} U[x(\pi)]\left({ }^{4}\right)$.

Choose a sequence of positive numbers $e_{n} \rightarrow 0$. It is clear that one can obtain a sequence $\pi_{n}$ such that $\pi_{n+1} \geqq \pi_{n}$ and for $\pi \geqq \pi_{n}\left\|x(\pi)-x\left(\pi_{n}\right)\right\| \leqq e_{n}$. Let $x^{\prime}\left(\pi_{n}\right)$ be one of the elements of $x\left(\pi_{n}\right)$. As $X$ is sequentially complete there will exist an $x \varepsilon X$ such that $x=\lim _{n} x^{\prime}\left(\pi_{n}\right)$. But then if $\pi \geqq \pi_{n},\|x-x(\pi)\| \leqq 2 e_{n}$ and likewise $\|U(x)-U[x(\pi)]\| \leqq\|U\| \cdot 2 e_{n}$. Hence $x=\lim _{\pi} x(\pi)$ and $U(x)$ $=\lim _{\pi} U[x(\pi)]$.

\subsection{Theorem. If $\phi(t) \varepsilon L^{p}(\alpha)$ and $\dot{x} \varepsilon V^{q}(X, \Gamma)$, then $\int \phi d x$ exists.}

Given $e>0$, there exists $\pi_{e}$ such that if $\pi \geqq \pi_{e}$ then $\sum_{\pi}\left|\phi\left(t_{i}\right)\right| p\left|\tau_{i}\right|<\infty$ and $\left|\sum_{x}\right| \phi\left(t_{i}\right)-\phi\left(t_{j}^{i}\right)|p| \tau_{i}|| \leqq e$ for all $t_{i} \varepsilon \tau_{i} \varepsilon \pi$ and all $t_{j}^{i} \varepsilon \tau_{j}^{i} \varepsilon \pi_{e}$ where $\tau_{i} \subset \tau_{j}^{i}$. Then

(3) If $B$ is a subset of $X,\|B\|=$ L.U.B. [ $\|x\| \| x \varepsilon B]$.

(4) Compare with Moore and Smith [20, p. 106]. 


$$
\left|\sum_{n}^{\infty}\right| \phi\left(t_{i}\right) \bar{x}\left[x\left(\tau_{i}\right)\right]|| \leqq\|\dot{x}\| \cdot\|\bar{x}\| \cdot\left[\sum_{n}^{\infty}\left|\phi\left(t_{i}\right)\right| p\left|\tau_{i}\right|\right]^{1 / p}
$$

and therefore approaches zero uniformly in the unit sphere of $\Gamma$ as $n \neq \infty$. It follows that $\sum_{\pi} \phi\left(t_{i}\right) x\left(\tau_{i}\right)$ is unconditionally convergent. Further if $\pi \geqq \pi_{e}$

$$
\begin{aligned}
\left\|\sum_{\pi} \phi\left(t_{i}\right) x\left(\tau_{i}\right)-\sum_{\pi_{e}} \phi\left(t_{j}\right) x\left(\tau_{j}\right)\right\| & =\underset{\dot{x} \varepsilon \dot{\Gamma},\|\dot{x}\|=1}{\text { L.U.B. }}\left|\sum_{\boldsymbol{\pi}}\left(\phi\left(t_{i}\right)-\phi\left(t_{j}^{i}\right)\right) \bar{x}\left[x\left(\tau_{i}\right)\right]\right| \\
& \leqq\|\dot{x}\| \cdot\left[\sum_{\pi}\left|\phi\left(t_{i}\right)-\phi\left(t_{j}^{i}\right)\right|^{p}\left|\tau_{i}\right|\right] \leqq\|\dot{x}\| \cdot e .
\end{aligned}
$$

Hence $\sum_{\pi} \phi\left(t_{i}\right) x\left(\tau_{i}\right)$ is a fundamental $\pi$-sequence and by Lemma 2.2 $\lim _{\pi} \sum_{\pi} \phi\left(t_{i}\right) x\left(\tau_{i}\right)=\int \phi d x$ exists.

2.4. Theorem. L.U.B. [ $\left.\left\|\int \phi d x\right\|\left\|\phi(t) \varepsilon L^{p}(\alpha),\right\| \phi \|=1\right]=\|\dot{x}\|$.

Since $\bar{x}(\dot{x}) \varepsilon V^{q}(R)\left(\bar{x}(\dot{x})\right.$ will be used to indicate the function $\left.\bar{x}[x(\tau)] \varepsilon V^{q}(R)\right)$

$$
\underset{\|\phi\|=1}{\operatorname{L.U.B.}}\left|\bar{x} \int \phi d x\right|=\underset{\|\phi\|=1}{\text { L.U.B. }}\left|\int \phi d \bar{x}(x)\right|=\|\bar{x}(\dot{x})\| \text {. }
$$

Therefore

$$
\begin{aligned}
\underset{\|\phi\|=1}{\text { L.U.B. }}\left\|\int \phi d x\right\| & =\text { L.U.B. }\left[\left|\bar{x} \int \phi d x\right| \mid \bar{x} \dot{\varepsilon} \Gamma,\|\bar{x}\|=1,\|\phi\|=1\right] \\
& =\text { L.U.B. }[\|\bar{x}(\dot{x})\| \mid \bar{x} \varepsilon \Gamma,\|\bar{x}\|=1]=\|\dot{x}\| .
\end{aligned}
$$

One can likewise define this integral on any measurable set. We designate the so-defined integral on $\tau$ by $\int_{\tau} \phi d x$. $\int_{\tau} \phi d x$ is clearly an additive set function on $\mathcal{T}$. Since by Theorem $2.4\left\|\int_{\tau} \phi d x\right\| \leqq\|\dot{x}\| \cdot\left[\int_{\tau}|\phi|^{p} d \alpha\right]^{1 / p}$, it follows that it is absolutely continuous and consequently completely additive.

2.5. TheOREM. If $\phi(t) \varepsilon L^{p}(\alpha), \alpha(T)<\infty$, and $\dot{x} \varepsilon V^{q}(X, \Gamma)$ is such that $x(\tau)=\int_{\tau}^{\prime} y(t) d \alpha$, then $\int \phi d x=\int^{\prime} \phi y d \alpha$ where $\int^{\prime}$ are both either Dunford integrals with $y(t) \& \mathcal{L}_{0}^{\mathfrak{a}}(E)[X, \Gamma][7]$ or Birkhoff integrals [3].

Suppose that $y(t) \varepsilon \mathcal{L}_{0}^{q}(E)[X, \Gamma]$; then $\bar{x}(x(\tau))=\int_{\tau} \bar{x}(y) d \alpha$ for every $\bar{x} \varepsilon \Gamma$. It follows from the similar theorem in real variables that

$$
\bar{x}\left[\int_{\tau} \phi d x\right]=\int_{\tau} \phi d \bar{x}(x)=\int_{\tau} \phi \bar{x}(y) d \alpha .
$$

This is equivalent to the statement that $\phi(t) \cdot y(t) \varepsilon \mathcal{L}_{0}^{1}(E)[X, \Gamma]$ and $\int \phi d x=\int^{\prime} \phi y d \alpha$.

On the other hand, suppose $y(t)$ is Birkhoff integrable to the value $x(\tau)$. Then let $\phi_{n}(t)=\phi(t)$ if $|\phi(t)| \leqq n$ and vanish elsewhere. $\phi_{n}(t) y(t)$ differs from 
$\phi(y) t(y)$ on a set whose measure approaches zero as $n \rightarrow \infty$. Since $\phi_{n}(t)$ is bounded, $\phi_{n}(t) \cdot y(t)$ is Birkhoff integrable [3, p. 369, Theorem 17]. Moreover it is integrable to the same value as the above Dunford integral so that $\int_{\tau} \phi_{n} y d \alpha=\int_{\tau} \phi_{n} d x$. By Theorem 2.4,

$$
\left\|\int_{\tau} \phi_{n} y d \alpha\right\|=\left\|\int_{\tau} \phi_{n} d x\right\| \leqq\|\dot{x}\| \cdot\left[\int_{\tau}\left|\phi_{n}\right|^{p} d \alpha\right]^{1 / p} \leqq\|\dot{x}\| \cdot\left[\int_{\tau}|\phi|^{p} d \alpha\right]^{1 / p} .
$$

The integrals $\int_{\tau} \phi_{n} y d \alpha$ are therefore uniformly absolutely continuous. By a theorem due to the author [23, Theorem 6.2] $\phi(t) \cdot y(t)$ is Birkhoff integrable and

$$
\int^{\prime} \phi y d \alpha=\lim _{n} \int \phi_{n} d x=\int \phi d x .
$$

We will next consider $\mathcal{T}$-measurable functions $\phi(t)$ either bounded or essentially bounded relative to a measure $\alpha . x(\tau) \varepsilon V^{1}(X, \Gamma)$ is defined on all $\tau \varepsilon \mathcal{T}$ and in the latter case vanishes on the null sets of $\alpha$. For convenience we will limit ourselves to the former case.

2.6. Definition. For a bounded function $\phi(t)$ and $\dot{x} \varepsilon V^{1}(X, \Gamma)$, we define

$$
\int \phi d x=\lim _{\pi} \sum_{\pi} \phi\left(t_{i}\right) x\left(\tau_{i}\right)
$$

whenever for $t_{i}$ an arbitrary element of $\tau_{i}$ this limit exists.

When $\int \phi d x$ exists by both Definition 2.1 and Definition 2.6, the value in each case is the same.

The following two theorems are special cases of a theorem due to Gowurin [13, pp. 265-266]. We omit their proofs.

2.7. Theorem. If $\phi(t)$ is bounded and $\dot{x} \varepsilon V^{1}(X, \Gamma)$, then $\int \phi d x$ exists.

2.8. Theorem. L.U.B. $\left[\left\|\int \phi d x\right\| \||\phi(t)| \leqq 1\right]=\|\dot{x}\|$.

It is unlikely that much can be said about the differentiation of $\dot{x} \varepsilon V^{q}(X, \bar{X})$ for $1 \leqq q<\infty$. Pettis has constructed an $\dot{x} \varepsilon V^{2}{ }_{c}\left(L^{2}, L^{2}\right)[22$, Example 9.4] which has no pseudo-derivative [22, p. 300]. In $\$ 5$ we demonstrate that $\dot{x} \varepsilon V^{\infty}{ }_{c}(X)$ ( $X$ arbitrary) and $\dot{x} \varepsilon V^{\infty}(X)$ ( $X$ separable and regular) for $T=\sum \tau_{i}\left(\left|\tau_{i}\right|<\infty\right)$ can be expressed as the Birkhoff integral of a function on $T$ to $X$.

We wish finally to consider an integral for functions $\phi(t) \varepsilon C$. In this connection Gelfand [12, pp. 246-253] has introduced the abstractly valued function classes $V(X)$ and $V_{c}(X) . V(X)$ is the class of all functions $x(t)$ on $(0,1)$ to $X$ for which $\bar{x}[x(t)]$ is of bounded variation and continuous on the left, while $V_{c}(X)$ is the subclass of $V(X)$ for which the set 


$$
\left[\sum\left(x\left(t_{i}\right)-x\left(t_{i}^{\prime}\right)\right) \mid\left(t_{i}, t_{i}^{\prime}\right) \text { disjoint intervals }\right]
$$

is compact. The L.U.B. [variation of $\bar{x}[x(t)] \mid\|\bar{x}\|=1$ ] exists and can be defined to be the norm $\|\dot{x}\|$ for elements of $V(X)$. It is easily shown that $V(X)$ is a Banach space having $V_{c}(X)$ as a closed linear subspace.

2.9. Definition. For functions $\phi(t) \varepsilon C$ and $\dot{x} \varepsilon V(X)$ we define

$$
\int \phi d x=\lim _{|\pi| \rightarrow 0} \sum_{\pi} \phi\left(t_{i}^{\prime}\right)\left[x\left(t_{i}\right)-x\left(t_{i-1}\right)\right] \quad(\pi \text { of type } 3)
$$

whenever for $t_{i}^{\prime}$ an arbitrary element of $\left(t_{i}, t_{i-1}\right)$ the limit exists.

2.10. ThEOREM. If $\phi(t) \varepsilon C$ and $\dot{x} \varepsilon V(X)$, then $\int \phi d x$ exists and L.U.B. $\left[\left\|\int \phi d x\right\||| \phi(t) \mid \leqq 1\right]=\|\dot{x}\|$.

This theorem has likewise been proven by Gowurin [13]. It is clear that $\bar{x}\left[\int \phi d x\right]=\int \phi d \bar{x}(x)$ so that this integral when it exists is equal to the integral defined by Gelfand [12, pp. 259-260].

3. On conditionally compact sets in a Banach space. In this section we will consider two different characterizations of conditionally compact sets in a Banach space $X$. The first is given in terms of a determining manifold $\Gamma$, while the second involves the notion of a generalized base.

3.1. Theorem. A necessary and sufficient condition that the set $S \equiv[x]$ be conditionally compact is that both L.U.B. $[|\bar{x}(x)| \mid x \varepsilon S]<\infty$ for each $\bar{x} \varepsilon \Gamma$ and $U(\bar{x})=\bar{x}(x)$ on $\Gamma$ to $M_{S}{ }^{(5)}$ be completely continuous.

Let $x_{n}$ be any denumerable subset of $S$. Its linear closed extension $Y$ is a separable Banach space. Let $\Gamma_{1}$ be the set of elements of $\Gamma$ considered as members of the conjugate space of $Y . \Gamma_{1}$ is clearly a determining manifold in the conjugate space of $Y$. The unit sphere of the conjugate space of a separable Banach space is a compact metric space in its weak topology [1, p. 186.]. Hence $\Gamma_{1}$ contains a denumerable subset $\left\{\bar{x}_{p}\right\}$ which is weakly dense in $\Gamma_{1}$. The linear transformation $V(x)=\bar{x}_{p}(x)$ on $Y$ to $m$ defines an equivalence. It is therefore sufficient to show that the set $\left\{\bar{x}_{p}\left(x_{n}\right)\right\}$ is conditionally compact in $m$. By the diagonal procedure we can obtain an $n$-subsequence $\bar{x}_{p}\left(x_{n^{\prime}}\right)$ such that $\lim _{n^{\prime}} \bar{x}_{p}\left(x_{n^{\prime}}\right)$ exists for every $p$. Moreover this limit exists uniformly in $p$. For if the contrary were true there would exist a $p$-subsequence having no subsequence for which the limit existed uniformly. As $\left\|\bar{x}_{p}\right\| \leqq 1$ and as $U$ is completely continuous, this $p$-subsequence would have a subsequence $p^{\prime}$ for which $\lim _{p^{\prime}} \bar{x}_{p^{\prime}}\left(x_{n^{\prime}}\right)$ exists uniformly in $p^{\prime}$ which gives a contradiction.

To prove the necessity we notice that the closed linear extension $Y$ of $S$ is a separable Banach space. Hence every bounded sequence of functionals bers.

(5) We remind the reader that $M_{S}$ is the space of bounded functions $\bar{x}(x)$ on $S$ to real num- 
on $Y$ contains a weakly convergent subsequence [1, p. 123, Theorem 3$]$. Since the subsequence is uniformly bounded in their norms the functions are equicontinuous and therefore converge uniformly on a compact set. The same is true for every bounded sequence of functionals on $X$ as we are concerned only with their values on $Y$. It follows that $U(\bar{x})$ is completely continuous.

Gelfand has proved the following corollary for the case $\Gamma=\bar{X}[12$, p. 268]. It should be pointed out that the corollary is not true for non-separable $X$ as was stated by Gelfand. In his argument he falsely assumed that the functionals of a weakly convergent sequence of functionals of a closed linear subspace of $X$ could be extended so that the sequence converged weakly on $X$ (see 7.5).

3.2. Corollary. A necessary and sufficient condition for a subset $S$ of a separable Banach space $X$ to be conditionally compact is that all weakly convergent sequences of functionals of $\Gamma$ on $X$ converge uniformly on $S$.

Every bounded sequence of functionals on $X$ contains a weakly convergent subsequence $[1$, p. 123 , Theorem 3$]$. By hypothesis this sequence converges uniformly on $S$ and hence the transformation $U(\bar{x})=\bar{x}(x)$ on $\Gamma$ to $M_{S}$ is completely continuous. By Theorem $3.1 S$ is conditionally compact. The necessity argument is similar to that used in Theorem 3.1.

The following lemma will permit us to prove that the corollary can not be extended to non-separable spaces even if, in its statement, $\Gamma$ is replaced by $\bar{X}$. We now suppose $T$ to be the class of all positive integers $t$ and $\mathcal{T}$ the family of all subsets $\tau$ of $T$.

3.3. Lемма. If $\beta^{n}(\tau)$ are bounded and finitely additive set functions on $\mathcal{T}$ to real numbers, and if $\lim _{n} \beta^{n}(\tau)=0$ for all $\tau \varepsilon \mathcal{E}$, then $\lim _{n} \sum_{t}\left|\beta^{n}(t)\right|=0$.

Suppose the lemma to be false. Then there exists an $e>0$ such that $\lim \sup _{n} \sum_{t}\left|\beta^{n}(t)\right|>e$. Now $\beta^{n}(t) \rightarrow 0$ as $n \rightarrow \infty$. Hence we can choose two increasing sequences of integers $n_{i}, N_{i}$ such that $\sum_{N_{i}+1}^{N_{i+1}-1}\left|\beta^{n_{i}}(t)\right| \geqq e$ and $\sum_{1}^{N_{i}-1}\left|\beta^{n_{i}}(t)\right|+\sum_{N_{i+1}}^{\infty}\left|\beta^{n_{i}}(t)\right|<e / 8$. Let us consider for the moment as a primary block some subset $\tau_{i}$ of $N_{i} \leqq t<N_{i+1}$ for which $\left|\beta^{n_{i}}\left(\tau_{i}\right)\right|>e / 2$. $\sum \tau_{i}$ is then divided into a denumerable set of disjoint blocks. Since a denumerable set has an infinite number of disjoint denumerable subsets and since $\beta^{n}(\tau)$ is bounded, there will exist a denumerable subset of blocks $\pi_{1}$ such that, on any of its subsets $\pi,\left|\beta^{n_{1}}(\pi)\right| \leqq e / 8$. The same argument gives a denumerable subset $\pi_{2}$ of $\pi_{1}$ such that, on any of its subsets $\pi,\left|\beta^{n_{2}}(\pi)\right| \leqq e / 8$. Likewise we can find a denumerable subset $\pi_{p}$ of $\pi_{p-1}$ such that on any of its subsets $\pi$, $\left|\beta^{n_{p}}(\pi)\right| \leqq e / 8$. Clearly $\tau_{p} \notin \pi_{p}$. Let $\pi_{0}$ consist of the $n$th block of $\pi_{n}$ for all $n$. If $\pi_{0}$ contains the block $\tau_{k}$, then there exists $q_{k} \geqq k$ such that

$$
\beta^{n_{k}}\left(\pi_{0}\right)=\sum_{i=1}^{q_{k}} \beta^{n_{k}}\left(\tau_{i} \cdot \pi_{0}\right)+\beta^{n_{k}}\left(\pi_{k} \cdot \pi_{0}\right) .
$$


Therefore

$$
\left|\beta^{n_{k}}\left(\pi_{0}\right)\right| \geqq\left|\beta^{n_{k}}\left(\tau_{k}\right)\right|-e / 8-e / 8 \geqq e / 4 \text {. }
$$

Since $\pi_{0}$ contains a denumerable number of such blocks, $\beta^{n}\left(\pi_{0}\right)$ does not approach 0 , which is contrary to our hypothesis.

As Hildebrandt [14] has shown, to every $\bar{x} \varepsilon \bar{m}$ there corresponds a unique additive bounded set function $\beta(\tau)$ on all sets of integers such that for all $x \varepsilon m, \bar{x}(x)=\int_{T} x(t) d \beta$. If $\bar{x}_{n}(x)=\int_{T} x(t) d \beta^{n}$ converge weakly to zero on $m$, then $\beta^{n}(\tau) \rightarrow 0$ for all $\tau \varepsilon \mathcal{T}$. We therefore have the following

3.4. CoRollaRy. If $\bar{x}_{n}(x)=\int_{T} x(t) d \beta^{n}$ converge weakly to zero on $m$, then $\sum_{t}\left|\beta^{n}(t)\right| \rightarrow 0$ as $n \rightarrow \infty$.

3.5. ExAmple. Let $S$ be the set of unit vectors $x_{p}$ in $m$. If $\bar{x}_{n}$ converges weakly to $\bar{x}_{0}$, then $\bar{y}_{n}=\bar{x}_{n}-\bar{x}_{0}$ converges weakly to zero. As above $\bar{y}_{n}(x)$ $=\int_{T} x(t) d \beta^{n}$ and $\sum_{t}\left|\beta^{n}(t)\right| \rightarrow 0$ as $n \rightarrow \infty$. Therefore $\bar{y}_{n}\left(x_{p}\right)=\beta^{n}(p) \rightarrow 0$ uniformly in $p$. In other words, $\bar{x}_{n}\left(x_{p}\right) \rightarrow \bar{x}_{0}\left(x_{p}\right)$ uniformly in $S$.

3.6. Theorem. If $U$ is additive and homogeneous on $X$ to $Y$ and $\bar{U}$ is completely continuous on $\Gamma$ to $\bar{X}$, then $U$ is completely continuous on $X$ to $Y$.

As Dunford [7, p. 317, Theorem 18] has shown, this hypothesis is sufficient to make $U$ a linear transformation on $X$ to $Y$. Let $S$ be the image under $U$ of $X_{1}$, the unit sphere of $X . \bar{y}(U(x))$ is then a linear transformation on $\Gamma$ to $M_{X_{1}}$. Given any sequence $\left\{\bar{y}_{n}\right\}$ in the unit sphere of $\Gamma$, there exists a subsequence $n^{\prime}$ such that $\bar{U}\left(\bar{y}_{n^{\prime}}\right)$ converges in $\bar{X}$. Hence $\bar{y}_{n^{\prime}}(U(x))=\bar{U}\left(\bar{y}_{n^{\prime}}\right)(x)$ converges in $M_{X_{1}}$. We can now apply Theorem 3.1 with $S=U\left(X_{1}\right) . S$ is conditionally compact and hence $U$ is completely continuous.

We will now give a second characterization of conditionally compact sets in a Banach space $\left.{ }^{6}\right) . \Pi$ will be a general range of elements $\pi$ transitive and compositive with respect to the relation $\geqq . U_{\pi}$ will be a set of completely continuous transformations on $X$ to $X$ defined on $\Pi$ with the properties: (1) For every $x \varepsilon X, \lim _{\pi} U_{\pi}(x)$ exists and is equal to $x$. (2) There exists a positive number $M$ such that $\left\|U_{\pi}\right\| \leqq M$ for all $\pi \varepsilon \Pi$. When the $U_{\pi}$ are in addition degenerate $\left({ }^{7}\right)$, such a class of transformations is called a generalized base of $X$.

3.7. TheOREM. Necessary and sufficient conditions that a set $S \subset X$ be conditionally compact are

(1) L.U.B. $[\|x\| \| x \varepsilon S]<\infty$,

(2) $\lim _{\pi}\left\|U_{\pi}(x)-x\right\|=0$ uniformly in $S$.

If we suppose $S$ to be conditionally compact, then given $e>0$, there exists

${ }^{(6)}$ Dr. T. H. Hildbrandt suggested Theorem 3.7 as a generalization of the author's application of it to $L^{p}$.

( ${ }^{7}$ ) A degenerate transformation on $X$ to $Y$ is a linear transformation on $X$ to a finite dimensional subspace of $Y$. 
$x_{1}, x_{2}, \cdots, x_{n} \subset X$ such that for any $x \varepsilon S$ there is a $k$ for which $\left\|x-x_{k}\right\|<e$. For the set $x_{1}, x_{2}, \cdots, x_{n}$ there exists a $\pi_{e}$ such that if $\pi \geqq \pi_{e}$, then $\left\|U_{\pi}\left(x_{k}\right)-x_{k}\right\| \leqq e$. Therefore if $x \varepsilon S$ and $\pi \geqq \pi_{e}$,

$$
\left\|U_{\pi}(x)-x\right\| \leqq\left\|U_{\pi}\left(x-x_{k}\right)\right\|+\left\|U_{\pi}\left(x_{k}\right)-x_{k}\right\|+\left\|x_{k}-x\right\| \leqq e(2+M),
$$

which proves the necessity.

The sufficiency argument is as follows: Given $e>0$, there exists $\pi_{e}$ such that $\left\|U_{\pi_{e}}(x)-x\right\|<e / 3$ for all $x \varepsilon S$. As $U_{\pi_{e}}$ is completely continuous and as L.U.B. [ $\|x\| \| x \varepsilon S]<\infty$, it follows that there exist $x_{1}, x_{2}, \cdots, x_{n} \subset S$ such that for any $x \in S$ there is a $k$ for which $\left\|U_{x_{e}}(x)-U_{\pi_{e}}\left(x_{k}\right)\right\| \leqq e / 3$. Therefore

$$
\left\|x-x_{k}\right\| \leqq\left\|x-U_{\pi_{e}}(x)\right\|+\left\|U_{\pi_{e}}\left(x-x_{k}\right)\right\|+\left\|U_{\pi_{e}}\left(x_{k}\right)-x_{k}\right\| \leqq e .
$$

$S$ is therefore totally bounded or, its equivalent, conditionally compact.

Theorem 3.7 gives a characterization of conditionally compact sets in $X$ which contains as a special case that given by Kolmogoroff [18], Tamarkin [26], and Tulajkov [27] for $L^{p}(\alpha)$ where $T=(0,1), \alpha$ is the Lebesgue measure, and $1 \leqq p<\infty$. In this case $\Pi$ is the set of integers and

$$
U_{n}(\phi)=\frac{n}{2} \int_{t-1 / n}^{t+1 / n} \phi(s) d s
$$

For $1<p<\infty,\left[U_{n}(\phi) \mid\|\phi\| \leqq 1\right]$ is uniformly bounded and equi-absolutely continuous, and therefore is conditionally compact in $L^{p}$. For $p=1$, $\left[U_{n}(\phi) \mid\|\phi\| \leqq 1\right]$ is of uniform bounded variation, and therefore is conditionally compact in $L$. Finally $\left\|U_{n}\right\| \leqq 1$ for $1 \leqq p<\infty$. The conclusions of the theorem are consequently valid.

Theorem 3.7 can also be applied to the spaces $L^{p}(\alpha)(1 \leqq p \leqq \infty)$ where $T$ is an abstract class of elements. For $1 \leqq p<\infty$, let $\pi$ be of type 1 and contain only a finite number of disjoint measurable sets $\left(\tau_{1}, \tau_{2}, \cdots, \tau_{n}\right) . \chi_{\tau}$ will denote the characteristic function of the set $\tau$. Finally we define $U_{\pi}$ on $L^{p}(\alpha)$ to $L^{p}(\alpha)$ to be

$$
\dot{U_{\pi}(\phi)}=\sum_{\pi} \frac{\int_{\tau_{i} \phi}}{\left|\tau_{i}\right|} \chi_{\tau_{i}} .
$$

For $p=\infty$, let $\pi$ be of type 2 and contain the disjoint measurable sets $\left(\tau_{1}, \tau_{2}, \cdots, \tau_{n}\right)$. Then $U_{\pi}$ on $L^{\infty}(\alpha)$ to $L^{\infty}(\alpha)$ will be

$$
U_{\pi}(\phi)=\sum_{\pi} \frac{\int_{\sigma_{i} \phi}}{\left|\sigma_{i}\right|} \chi_{r_{i}}
$$

where $\sigma_{i}$ is some set of finite measure contained in $\tau_{i}$. The $U_{\pi}$ clearly define a generalized base for $L^{p}(\alpha)$.

4. Linear transformations. In the following discussion for $1 \leqq p<\infty, \pi$ will be of type 1 ; while for $p=\infty, \pi$ will be of type $2 . L^{p}(1 \leqq p<\infty)$ will be of the 
space $L^{p}(\alpha) . L^{\infty}$ will be either (a) the space of bounded $\tau$-measurable functions, or (b) the space of $\tau$-measurable functions essentially bounded relative to a measure $\alpha$ with $x(\tau) \varepsilon V^{1}(X, \Gamma)$ vanishing on null sets in the latter case. $1 / p+1 / q=1$.

4.1. THEOREM. The general form of the linear transformation $U(\phi)$ on $L^{p}$ $(1 \leqq p \leqq \infty)$ to $X$ is

$$
U(\phi)=\int \phi d x
$$

where $\dot{x} \varepsilon V^{q}(X, \Gamma)$ and $\|U\|=\|\dot{x}\|$.

If $\dot{x} \varepsilon V^{q}(X, \Gamma)$ then it follows from Theorems $2.3,2.4,2.7$, and 2.8 that $\int \phi d x$ is a linear transformation on $L^{p}$ to $X$ with $\|U\|=\|\dot{x}\|$.

To demonstrate the converse, let $\chi_{\tau}(t)$ be the characteristic function of $\tau \varepsilon \mathcal{\tau}$ for $|\tau|<\infty$. We define

$$
x(\tau)=U\left[\chi_{\tau}(t)\right] .
$$

$x(\tau)$ is obviously additive on sets $\tau$ of finite measure. For $p=1$,

$$
\begin{aligned}
\|U\| & =\text { L.U.B. }[|\bar{x}[U(\phi)]|=|\bar{U}(\bar{x})[\phi]| \mid\|\bar{x}\|=1,\|\phi\|=1] \\
& =\text { L.U.B. }\left[\left|\frac{\bar{U}(\bar{x})\left[\chi_{\tau}\right]}{|\tau|}\right||\|\bar{x}\|=1, \tau \varepsilon \tau,| \tau \mid<\infty\right] \\
& =\text { L.U.B. }\left[\frac{\|x(\tau)\|}{|\tau|}|\tau \varepsilon \tau,| \tau \mid<\infty\right] .
\end{aligned}
$$

Therefore $\dot{x} \varepsilon V^{\infty}(X)$ and $\|U\|=\|\dot{x}\|$. For $1<p<\infty$, we define, for a given $\pi$ and $\left\{a_{i}\right\} \& l^{p}, \phi(t)=a_{i}\left|\tau_{i}\right|^{-1 / p}$ when $t \varepsilon \tau_{i}$. Then

$$
\begin{aligned}
\|\phi\| & =\left\{\int|\phi|^{p} d \alpha\right\}^{1 / p}=\left\{\sum\left|a_{i}\right|^{p}\right\}^{1 / p}=\|a\|, \\
U(\phi) & =\sum_{\pi} \phi\left(t_{i}\right) x\left(\tau_{i}\right)=\sum_{\pi} \frac{a_{i} x\left(\tau_{i}\right)}{\left|\tau_{i}\right|^{1 / p}}
\end{aligned}
$$

Finally

$$
\begin{aligned}
\|U\|= & \text { L.U.B. }\left[\left|\sum_{\pi} \frac{a_{i} \bar{x}\left[x\left(\tau_{i}\right)\right]}{\left|\tau_{i}\right| 1 / p}\right| \mid\|\bar{x}\|=1, \bar{x} \varepsilon \Gamma, \pi, a \varepsilon l^{p},\|a\|=1\right] \\
& \text { L.U.B. }\left[\left\{\sum_{\pi} \frac{\left|\bar{x}\left[x\left(\tau_{i}\right)\right]\right|^{q}}{\left|\tau_{i}\right|^{q-1}}\right\}^{1 / q} \mid \pi, \bar{x} \varepsilon \Gamma,\|\bar{x}\|=1\right]=\|\dot{x}\| .
\end{aligned}
$$

Again $\dot{x} \varepsilon V^{q}(X, \Gamma)$ and $\|U\|=\|\dot{x}\|$. For $p=\infty$, 


$$
\begin{aligned}
\|U\| & =\text { L.U.B. }\left[\left|\sum_{\pi} \phi\left(t_{i}\right) \bar{x}\left[x\left(\tau_{i}\right)\right]\right||\bar{x} \varepsilon \Gamma,\|\bar{x}\|=1, \pi,| \phi\left(t_{i}\right) \mid \leqq 1\right] \\
& =\text { L.U.B. }\left[\sum_{\pi}\left|\bar{x}\left[x\left(\tau_{i}\right)\right]\right| \mid \bar{x} \varepsilon \Gamma,\|\bar{x}\|=1, \pi\right]=\|\dot{x}\|,
\end{aligned}
$$

$\dot{x} \varepsilon V^{1}(X, \Gamma)$. To each $\phi(t) \varepsilon L^{p}$ and $\pi$ we associate the multiple valued function $\phi_{\pi}(t)=\phi\left(\tau_{i}\right)$ for $t \varepsilon \tau_{i}$ where $t_{i} \varepsilon \tau_{i}$. Then $\phi=\lim _{\pi} \phi_{\pi}$ in $L^{p}$ and by Lemma 2.2 and Theorems 2.3 and 2.7

$$
U(\phi)=\lim _{\pi} U\left(\phi_{\pi}\right)=\int \phi d x .
$$

For $p=\infty$ Theorem 4.1 has been demonstrated by Gowurin [13, pp. 265266].

4.2. Corollary. $V^{q}(X, \Gamma)$ is equivalent to $V^{q}(X, \bar{X}), 1 \leqq p \leqq \infty$.

By Theorem 4.1, $V^{q}(X, \Gamma)$ is equivalent to the space of all linear transformations on $L^{p}$ to $X$ for all $\Gamma$.

4.3. THEOREM. The general form of the completely continuous transformation $U(\phi)$ on $L^{p}(1 \leqq p \leqq \infty)$ to $X$ is

$$
U(\phi)=\int \phi d x
$$

where $\dot{x} \varepsilon V_{c}^{q_{c}}(X, \Gamma)$ and $\|U\|=\|\dot{x}\|$.

This is an immediate consequence of Theorems 3.6 and 4.1.

4.4. Corollary. $V^{q}{ }_{c}(X, \Gamma)$ is equivalent to $V^{q}{ }_{c}(X, \bar{X}), 1 \leqq q \leqq \infty$.

4.5. THEOREM. The general form of the linear transformation $U(x)$ on $X$ to $V^{q}(R)(1<q<\infty)$ where $T=\sum_{i=1}^{\infty} \tau_{i}\left(\left|\tau_{i}\right|<\infty\right)$ is

$$
U(x)=\bar{x}(\tau)[x]
$$

where $\dot{\bar{x}} \varepsilon V^{q}(\bar{X}, X)$ and $\|U\|=\|\dot{\bar{x}}\|\left({ }^{8}\right)$.

It is clear that $\dot{\bar{x}} \varepsilon V^{q}(\bar{X}, X)$ defines such a transformation and that $\|U\|=\|\dot{\bar{x}}\|$. Conversely, if $U$ is linear on $X$ to $V^{q}(R)$, then its adjoint $\bar{U}$ defines a transformation on $V^{p}(R)$ or its equivalent $L^{p}(\alpha)$ to $\bar{X}$. By Theorem 4.1

$$
\bar{U}(\phi)=\int \phi d \bar{x}
$$

where $\phi \varepsilon L^{p}(\alpha)$, $\dot{\bar{x}} \varepsilon V^{q}(\bar{X}, X)$, and $\|\bar{U}\|=\|\dot{\bar{x}}\|$. Since $\phi[U(x)]=\int \phi d x(\bar{x})$ for every $\phi \varepsilon L^{p}(\alpha)$, it follows that $U(x)=\bar{x}(\tau)[x]$ and $\|U\|=\|\bar{U}\|=\|\dot{\bar{x}}\|$.

(8) Compare with Kantorovitch and Vulich [17, pp. 133-135]. 
4.6. THEOREM. The general form of the completely continuous transformation $U(x)$ on $X$ to $V^{q}(R)(1<q<\infty)$ where $T=\sum_{i=1}^{\infty} \tau_{i}\left(\left|\tau_{i}\right|<\infty\right)$ is

$$
U(x)=\bar{x}(\tau)[x]
$$

where $\dot{\bar{x}} \varepsilon V_{c}^{q}(\bar{X}, X)$ and $\|U\|=\|\dot{\bar{x}}\|$.

The argument used in Theorem 4.5 applies here if we note that $\bar{U}$ is necessarily completely continuous $[1$, p. 101 , Theorem 4$]$. The reference is now made to Theorem 4.3 instead of Theorem 4.1.

If $T=(0,1)$ and $\alpha$ is the Lebesgue measure, then the general form of the linear (or completely continuous) transformation on $X$ to $L^{q}(1<q<\infty)$ is

$$
U(x)=\frac{d}{d t} \bar{x}\left(I_{0}^{t}\right) \cdot[x]
$$

where $\dot{\bar{x}} \varepsilon V^{q}(\bar{X}, X)\left(\right.$ or $\left.V^{q} c(\bar{X}, X)\right),\|U\|=\|\dot{\bar{x}}\|$ and $\left.I_{0}^{t}\right)=(0, t)$. This is a slightly stronger result than that found by Bochner and Taylor [5, pp. 941-944, Theorems 8.1 and 8.4 ].

A linear transformation on $L^{p}$ to $L^{p^{\prime}}$, where $\alpha$ is the Lebesgue measure on $(0,1)$, is characterized by a function $K(s, \tau)$ for which

$$
\frac{d}{d t} \int_{0}^{1} \psi(s) K\left(s, I_{0}^{t}\right) d s \varepsilon L^{a}
$$

for every $\psi \varepsilon L^{q^{\prime}}\left(I^{t}{ }_{0}=(0, t)\right)$. If in addition the transformation is completely continuous, then $K(s, \tau)$ also satisfies the condition

$$
\left.\left.\lim _{h \rightarrow 0} \int_{0}^{1} \mid \frac{d}{d t} \int_{0}^{1} \psi(s) K\left(s, I_{0}^{t}\right) d s\right]_{t+h}-\frac{d}{d t} \int_{0}^{1} \psi(s) K\left(s, I_{0}^{t}\right) d s\right]_{t}^{q} d t=0
$$

uniformly for all $\psi \varepsilon L^{q^{\prime}}$.

We leave the proof of the following theorems to the reader. Except for the space $c_{0}$, the argument is a special case of the above. Gelfand has discussed the space $c\left[12\right.$, pp. 272-275]. It is convenient to denote the space $c_{0}$ by the symbol $l^{\infty}$.

4.7. THEOREM. The general form of the linear [or completely continuous] transformation $U(a)$ on $l^{p}(1 \leqq p \leqq \infty)$ to $X$ is

$$
U(a)=\sum a_{i} x_{i}
$$

where $\dot{x} \varepsilon v^{q}(X, \Gamma)\left[\right.$ or $\left.\dot{x} \varepsilon v^{q}{ }_{c}(X, \Gamma)\right]$ and $\|U\|=\|\dot{x}\|$.

4.8. Corollary. $v^{q}(X, \Gamma)\left[\right.$ or $\left.v_{c}^{q}(X, \Gamma)\right]$ is equivalent to $v^{q}(X, \bar{X})$ [or $\left.v_{c}^{q}(X, \bar{X})\right](1 \leqq q \leqq \infty)$.

4.9. COROLlaRY. If $X$ is either weakly complete or a separable conjugate space, then any linear transformation on $c_{0}$ to $X$ is completely continuous. 
$\dot{x} \varepsilon v^{1}(X, \bar{X})$ implies that $\dot{x} \varepsilon v^{1}{ }_{c}(X, \bar{X})$ according to a theorem of Orlicz [21, pp. 244-247] and Theorem 1.8. The conclusion follows from Theorem 4.7.

4.10. TheOREM. The general form of the linear [or completely continuous] transformation $U(x)$ on $X$ to $l^{q}(1 \leqq q<\infty)$ is

$$
U(x)=\left\{\bar{x}_{i}(x)\right\}
$$

where $\dot{\bar{x}} \varepsilon v^{q}(\bar{X}, X)\left[\right.$ or $\left.\dot{\bar{x}} \varepsilon v^{q}{ }_{c}(\bar{X}, X)\right]$ and $\|U\|=\|\dot{\bar{x}}\|$.

4.11. CoRollary. If $X$ is either weakly complete or a separable conjugate space, then any linear transformation on $X$ to $l^{1}$ is completely continuous.

We conclude this section with some considerations about linear transformations on $C$ to $X$. It follows from Theorem 2.10 that $U(\phi)=\int \phi d x$ where $\phi \varepsilon C$ and $\dot{x} \varepsilon V(X)$ is a linear transformation on $C$ to $X$ with $\|U\|=\|\dot{x}\|$. Gelfand $[12$, p. 283] has shown that the general form of a completely continuous transformation on $C$ to $X$ is

$$
U(\phi)=\int \phi d x
$$

where $\dot{x} \varepsilon V_{c}(X)$ and $\|\dot{x}\|=\|U\|$. When $X$ is weakly complete, Gelfand has shown this to be the general form of the linear transformation on $C$ to $X$ where now $\dot{x} \varepsilon V(X)$. It might be added that Gelfand's method will show this to be true for all conjugate Banach spaces $X$.

It is easy to give an example of a linear transformation on $C$ to $X$ which does not have this general form. Let $U$ be the identity transformation on $C$ to $C$ and suppose that it does have this form. Then $\phi(s)=U(\phi)=\int \phi(t) d \psi_{t}(s)$. As this holds for all $\phi \varepsilon C, \psi_{t}(s)=c(s>t)$ and $\psi_{t}(s)=1+c(s<t)$, which is contrary to $\psi_{t}(s) \varepsilon C$ for fixed $t$. Because of the above remark, this again shows that $C$ is not a conjugate space.

The following theorem gives a characterization for linear transformations on $C$ to $X$ :

4.12. Theorem. A necessary and sufficient condition that $U$ be a linear transformation on $C$ to $X$ is that there exist a sequence of step functions $\dot{x}_{n} \varepsilon V(X)$ such that $\lim _{n \rightarrow \infty}\left\|\dot{x}_{n}\right\|=\|U\|$ and

$$
U(\phi)=\lim _{n} \int \phi d x_{n}
$$

Making use of the Bernstein polynomials

$$
\phi(t)=\lim _{n \rightarrow \infty} \sum_{r=0}^{n} C_{r}^{n} \phi(r / n) t^{r}(1-t)^{n-r}
$$


in $C$. If we apply a device due to Hildebrandt and Schoenberg [15, p. 318], then

$$
U(\phi)=\lim _{n \rightarrow \infty} \sum_{r=0}^{n} \phi(r / n) U\left[C_{r}^{n} t^{r}(1-t)^{n-r}\right]=\lim _{n \rightarrow \infty} U_{n}(\phi)
$$

where $U_{n}(\phi)=\int \phi d x_{n}, x_{n}(t)$ being defined to have the jump $U\left[C_{r}^{n} t^{r}(1-t)^{n-r}\right]$ at $r / n$ and to be constant elsewhere. Now

$$
\left\|U_{n}(\phi)\right\|=\left\|U\left[\sum C_{r}^{n} \phi(r / n) t^{r}(1-t)^{n-r}\right]\right\| \leqq\|U\| \cdot\|\phi\| .
$$

Therefore $\left\|\dot{x}_{n}\right\|=\left\|U_{n}\right\| \leqq\|U\|$. In general, however, $\lim \inf _{n \rightarrow \infty}\left\|U_{n}\right\| \geqq\|U\|$ so that $\lim _{n \rightarrow \infty}\left\|\dot{x}_{n}\right\|=\|U\|$. Since $\dot{x}_{n}$ does define a linear transformation, the sufficiency argument is obvious $[1$, p. 80 , Theorem 5$]$.

5. Linear transformations on $L$. In this section we obtain representations by means of a kernel of the general completely continuous transformation and weakly completely continuous separable transformation on $L$ to an arbitrary Banach space $X$. By means of this result and a theorem due to Dunford and Pettis [9], we show that $U^{2}$ is completely continuous whenever $U$ on $L$ to $L$ is weakly completely continuous. Special cases of Theorems 5.3 and 5.4 have been proved by Gelfand [12]. More recently Dunford and Pettis [9] have obtained special cases of Theorems 5.3, 5.4, and 5.5.

In this and the following section, $L^{p}$ will be the space $L^{p}(\alpha)$ where $T$ is the sum of a denumerable number of sets $\tau \varepsilon \mathcal{\tau}$ of finite measure. $\pi$ will be defined as at the end of $\S 3 . x(t)$ on $T$ to $X$ will be said to be weakly measurable if $\bar{x}(x(t))$ is measurable for all $\bar{x} \varepsilon \bar{X}$. We define $B^{\infty}(X)$ to be the class of weakly measurable point functions $x(t)$ on $T$ to $X$ whose values are essentially contained in a separable conditionally weakly compact subspace of $X$. With norm

$$
\|\dot{x}\|=\text { ess. L.U.B.t }[\|x(t)\|],
$$

$B^{\infty}(X)$ is a Banach space. The set of functions $x(t) \varepsilon B^{\infty}(X)$ which take on a.e. a conditionally compact set of values will comprise the subspace $B^{\infty}{ }_{c}(X)$ of $B^{\infty}(X)$.

Integration with respect to a real valued measure function $\alpha$ will be realized by means of the Birkhoff integral [3]. Since $x(t)$ for $\dot{x} \varepsilon B^{\infty}(X)$ is a.e. contained in a separable subspace of $X, x(t)$ is integrable on all sets $\tau \varepsilon \mathcal{T}$ of finite measure [22, Theorems 1.1 and 5.3, Corollary 5.11].

5.1. Lemma. If $\dot{x} \varepsilon B^{\infty}(X)$, then

$$
\|\dot{x}\|=\text { L.U.B. }\left[\frac{\left\|\int_{\tau} x(t) d \alpha\right\|}{|\tau|}|0<| \tau \mid<\infty, \tau \varepsilon \tau\right] .
$$

Since $x(t)$ is essentially contained in a separable subspace $X^{\prime}$ of $X$, it is clear that there exists a denumerable set of linear functionals $\left\{\bar{x}_{n}\right\} \subset \bar{X}$ each 
of norm one such that L.U.B. $\left[\left|\bar{x}_{n}(x)\right|=\|x\| \mid n, x \varepsilon X^{\prime}\right]$. Therefore

$$
\begin{aligned}
A & =\text { L.U.B. }\left[\frac{\left\|\int_{\tau} x(t) d \alpha\right\|}{|\tau|}|0<| \tau \mid<\infty, \tau \varepsilon \mathcal{T}\right] \\
& =\text { L.U.B. }\left[\frac{\left|\int_{\tau} \bar{x}_{n}(x(t)) d \alpha\right|}{|\tau|}|0<| \tau \mid<\infty, \tau \varepsilon \mathcal{\tau}, n\right] .
\end{aligned}
$$

Let $\tau_{n}=\left[t|| \bar{x}_{n}(x(t)) \mid>A\right]$. Clearly $\left|\tau_{n}\right|=0 . \tau_{0}=\left[t \mid\|x(t)\|>A, x(t) \varepsilon X^{\prime}\right]$ is contained in $\sum \tau_{n}$ and hence is of measure zero. On the other hand for every $e>0$, there exists an $n$ such that ess. L.U.B. $t\left|\bar{x}_{n}(x(t))\right|>A-e$. It follows that ess. L.U.B. $t\|x(t)\|=A$.

5.2. Theorem. $V^{\infty}{ }_{c}(X)$ is equivalent to $B^{\infty}{ }_{c}(X)$. The equivalence is defined by $U(x(t))=x(\tau)=\int_{\tau} x(t) d \alpha$ on $B^{\infty}{ }_{c}(X)$ to $V^{\infty}{ }_{c}(X)$.

It follows from Lemma 5.1 that $U$ is an isometric transformation. By the definition of the Birkhoff integral, given any null set $\tau_{0}$, $S=[x(\tau) /|\tau||0<| \tau \mid<\infty, \tau \varepsilon \mathcal{\tau}]$ is contained in the convex extension of $\left[x(t) \mid t \varepsilon T-\tau_{0}\right]$. Therefore $S$ is conditionally compact. By Theorem 1.4,

$$
x(\tau)=U(x(t)) \varepsilon V^{\infty}{ }_{c}(X) .
$$

We next prove the converse. Let $x(\tau) \varepsilon V^{\infty}{ }_{c}(X) . \bar{x}(x(\tau))$ is then a completely additive set function on all measurable subsets of any set of finite measure. By the Radon-Nikodym theorem there exists $f_{\tilde{x}}(t) \varepsilon B^{\infty}(R)$ such that $\bar{x}(x(\tau))=\int_{\tau} f_{\bar{x}}(t) d \alpha$ for all $\tau \varepsilon \mathcal{G}$ of finite measure. As above this defines an isometric transformation $V$ on $B^{\infty}(R)$ to $V^{\infty}(R)$. By definition, $[\bar{x}(x(\tau)) \mid \bar{x} \varepsilon \bar{X},\|\bar{x}\| \leqq 1]$ is conditionally compact in $V^{\infty}(R)$. Therefore $P=\left[f_{\bar{x}}(t) \mid \bar{x} \varepsilon \bar{X},\|\bar{x}\| \leqq 1\right]$ is conditionally compact in $B^{\infty}(R)$. Defining $U_{x}$ as in $\S 3$, it follows from Theorem 3.7 that $\lim _{\pi} U_{\pi}\left(f_{\tilde{x}}(t)\right)=f_{\bar{x}}(t)$ uniformly in $P$ in the topology of $B^{\infty}(R)$.

$$
V\left(U_{\pi}\left(f_{\tilde{x}}(t)\right)\right)=\sum_{\pi} \frac{\bar{x}\left[x\left(\sigma_{i}\right)\right]}{\left|\sigma_{i}\right|} \cdot\left|\tau \cdot \tau_{i}\right| .
$$

Then

$$
\lim _{\approx} V\left(U_{\pi}\left(f_{\tilde{x}}(t)\right)\right)=V\left(f_{\tilde{x}}(t)\right)
$$

uniformly in $P$. This implies

$$
\lim _{\pi} \sum_{\pi} \frac{x\left(\sigma_{i}\right)}{\left|\sigma_{i}\right|}\left|\tau \cdot \tau_{i}\right|=x(\tau)
$$

in $V^{\infty}{ }_{c}(X)$. Define $x_{\pi}(t)=x\left(\sigma_{i}\right) /\left|\sigma_{i}\right|$ for $t \varepsilon \tau_{i}$; and $x_{\pi}(\tau)=\int x_{\pi}(t) d \alpha$. Then $x_{\pi}(t) \varepsilon B_{c}^{\infty}(X)$; 


$$
x_{\pi}(\tau)=\sum_{\pi} \frac{x\left(\sigma_{i}\right)}{\left|\sigma_{i}\right|}\left|\tau \cdot \tau_{i}\right|=U\left(x_{\pi}(t)\right) .
$$

By (1), given $e>0$, there exists a $\pi_{e}$ such that for $\pi_{1}, \pi_{2} \geqq \pi_{e},\left\|x_{\pi_{1}}(\tau)-x_{x_{2}}(\tau)\right\| v^{\infty}(X)$ $\leqq e$. According to Lemma 5.1, ess. L.U.B. $t\left\|x_{\pi_{1}}(t)-x_{\pi_{2}}(t)\right\| \leqq e$. Since $B^{\infty}{ }_{c}(X)$ is a Banach space, it follows from Lemma 2.2 that there exists an $x(t) \varepsilon B^{\infty}{ }_{c}(X)$ such that $\lim _{\pi} x_{\pi}(t)=x(t)$ in $B^{\infty}{ }_{c}(X)$. Then $x(\tau)=\lim _{\pi} U\left(x_{\pi}(t)\right)=U(x(t))$.

5.3. THEOREM. The general form of the completely continuous transformation $U$ on $L$ to $X$ is

$$
U(\phi)=\int \phi(t) x(t) d \alpha
$$

where $\dot{x} \varepsilon B^{\infty}{ }_{c}(X)$ and $\|U\|=\|\dot{x}\|$.

According to Theorem 4.3, $U(\phi)=\int \phi(t) d x$ where $x(\tau) \varepsilon V^{\infty}{ }_{c}(X)$ and $\|U\|=\|x(\tau)\|_{V^{\infty}(X)}$. By Theorem 5.2 there exists an $x(t) \varepsilon B^{\infty}{ }_{c}(X)$ such that $x(\tau)=\int_{\tau} x(t) d \alpha$ for all $\tau \varepsilon \mathcal{T}$ of finite measure and $\|x(t)\|_{B^{\infty}(X)}=\|x(\tau)\|_{V^{\infty}(X)}$ $=\|U\|$. Further by Theorem 2.5, $\int_{\tau} \phi(t) d x=\int_{\tau} \phi(t) x(t) d \alpha$ for all $\tau \varepsilon \mathcal{V}$ of finite measure. Now $T=\sum \tau_{i}$ where $\left|\tau_{i}\right|<\infty$ and $\tau_{i} \cdot \tau_{j}=0$ if $i \neq j$. Given $e>0$, one can obtain an unconditionally convergent sum of the type $\sum_{j} \phi\left(t_{j}\right) x\left(t_{j}\right)\left|\tau_{j}^{i}\right|$ $\left(\left(\tau_{j}^{i}\right)\right.$ is a subdivision of $\left.\tau_{i}, t_{j} \varepsilon \tau_{j}^{i}\right)$ which approximates $\int_{\tau_{i}} \phi(t) x(t) d \alpha$ to within $e / 2^{i}$ and such that each of its finite partial sums is within $e / 2^{i}$ of some $\int_{\tau} \phi(t) x(t) d \alpha$ for $\tau \subset \tau_{i}$. Since by Theorem 2.4, $\left\|\int_{\tau} \phi(t) x(t) d \alpha\right\| \leqq\|\dot{x}\| \int_{\tau}|\phi(t)| d \alpha$, it follows that the resulting subdivision of $T$ furnishes an unconditionally convergent sum which approximates $\int_{T} \phi(t) d x$ to within $e . \int_{T} \phi(t) x(t) d \alpha$ therefore exists and is equal to $\int_{T} \phi(t) d x$.

5.4. THEOREM. The general form of the weakly completely continuous separable transformation on $L$ to $X$ is

$$
U(\phi)=\int \phi(t) x(t) d \alpha
$$

where $\dot{x} \varepsilon B^{\infty}(X)$ and $\|U\|=\|\dot{x}\|$.

According to Theorem 4.1, $U(\phi)=\int \phi(t) d x$ where $x(\tau) \varepsilon V^{\infty}(X)$ and $\|U\|=\|x(\tau)\|_{V^{\infty}(X)}$. If $\chi_{\tau}(t)$ is the characteristic function of $\tau \varepsilon \mathcal{T}$ for $|\tau|<\infty$, then $x(\tau)=U\left(\chi_{\tau}(t)\right)$. As $U$ is weakly completely continuous and separable, it is clear that $S \equiv[x(\tau) /|\tau||0<| \tau \mid<\infty]$ is conditionally weakly compact and is contained in a separable linear closed subspace $Y$ of $X$. Hence there exists a sequence $\left\{\bar{x}_{n}\right\} \subset \bar{X}$ which when considered as elements of $\bar{Y}$ are dense in the unit sphere of a determining manifold in $\bar{Y}$. By the Radon-Nikodym theorem there exists for each $\bar{x} \varepsilon \bar{X}$ an $f_{\bar{x}}(t) \varepsilon B^{\infty}(R)$ such that $\bar{x}(x(\tau))=\int_{\tau} f_{\bar{x}}(t) d \alpha$ for all $\tau \varepsilon \mathcal{T}$ of finite measure. As in Theorem 5.2, let $\pi$ be a finite subdivision 
of $T$ into disjoint measurable sets $\left(\tau_{1}, \tau_{2}, \cdots, \tau_{n}\right)$, let $\sigma_{i} \subset \tau_{i}$ such that $0<\left|\sigma_{i}\right|<\infty$, and let $x_{\pi}(t)=x\left(\sigma_{i}\right) /\left|\sigma_{i}\right|$ for $t \varepsilon \tau_{i}$. Then for every $\bar{x} \varepsilon \bar{X}$, $\lim _{\pi} \bar{x}\left(x_{\pi}(t)\right)=f_{\bar{x}}(t)$ in $B^{\infty}(R)$. There will therefore exist a set $\left\{\pi_{n}\right\}$ such that $\left|\bar{x}_{i}\left(x_{\pi_{n}}(t)\right)-f_{\bar{x}_{i}}(t)\right|<1 / n$ on $T-\sigma_{n}\left(\left|\sigma_{n}\right|=0\right)$ for all $i \leqq n$. Hence for each $i$, $\bar{x}_{i}\left(x_{\pi_{n}}(t)\right) \rightarrow f_{\bar{x}_{i}}(t)$ uniformly on $T-\sigma_{0}$ where $\sigma_{0}=\sum \sigma_{n}\left(\left|\sigma_{0}\right|=0\right)$. For a given $t$, $x_{\pi_{n}}(t)$ is contained in $S$. A subsequence will therefore converge weakly to an element of $Y[1$, p. 134, Theorem 2]. We arbitrarily define $x(t)$ to be the weak limit of one such subsequence. Clearly $\bar{x}_{i}(x(t))=\lim _{n} \bar{x}_{i}\left(x_{\pi_{n}}(t)\right)=f_{\bar{x}_{i}}(t)$ on $T-\sigma_{0}$. As $\left|\bar{x}_{i}(x(t))\right| \leqq U$ on $T-\sigma_{0}$, and $x(t) \varepsilon Y$, it follows that $\|x(t)\| \leqq U$ on $T-\sigma_{0}$. Further $\bar{x}_{i}(x(t))$ is measurable, $Y$ is separable, and the sequence $\bar{x}_{i}$ is dense in the unit sphere of a determining manifold in $\bar{Y}$ if the $\bar{x}_{i}$ are considered as elements of $\bar{Y}$. From this one can easily show that $x(t)$ is weakly measurable. As $x(t)$ is contained in the sequential weak closure of $S$, $[x(t) \mid t \varepsilon T]$ is conditionally weakly compact $\left({ }^{9}\right)$. Therefore $x(t) \varepsilon B^{\infty}(X)$. Now $\bar{x}_{i}(x(\tau))=\int_{\tau} f_{\bar{x}_{i}}(t) d \alpha=\int_{\tau} \bar{x}_{i}(x(t)) d \alpha$ for all $\tau \varepsilon \mathcal{T}$ of finite measure. As $\left\{\bar{x}_{i}\right\}$ is total in $Y$, it follows that $x(\tau)=\int_{\tau} x(t) d \alpha$ for all $\tau$ of finite measure [23, Theorem 5.3]. By Lemma 5.1 $\|x(t)\|_{B^{\infty}(X)}=\|x(\tau)\|_{V^{\infty}(X)}=\|U\|$. The remainder of the argument is identical to that used in Theorem 5.3.

We remark that Theorem 5.4 is applicable to any separable linear transformation on $L$ to a regular Banach space since the unit sphere of a regular space is weakly compact.

In the following theorem and corollary, $T$ need not be the sum of a denumerable number of sets $\tau \varepsilon \mathcal{\tau}$ of finite measure.

5.5 THEOREM. If $U$ is a weakly completely continuous transformation on $L$ to an arbitrary Banach space $X$, then $U$ takes conditionally weakly compact sets into conditionally compact sets.

It is sufficient to show that for any conditionally weakly compact sequence $\left\{\phi_{n}\right\},\left\{U\left(\phi_{n}\right)\right\}$ is conditionally compact. The sequence $\left\{\phi_{n}\right\}$ is contained in a separable subspace $L^{\prime}$ of $L$ essentially defined on a class $T^{\prime} \subset T$ which is the sum of a denumerable number of sets of finite measure $\left({ }^{10}\right)$. Let $U^{\prime}$ on $L^{\prime}$ to $X$ be identical with $U$ on $L^{\prime}$. As $L^{\prime}$ is separable, $U^{\prime}$ is a separable weakly completely continuous transformation on $L^{\prime}$ to $X$. Theorem 5.4 is applicable, and hence by a theorem due to Dunford and Pettis $[9$, p. 547, Theorem 4] $U^{\prime}$ takes conditionally weakly compact sets into conditionally compact sets. Since $\left\{\phi_{n}\right\}$ is conditionally weakly compact in $L^{\prime}$, this concludes the proof.

(9) W. L. Chmoulyan has shown that the weak sequential closure of a weakly compact subset of a Banach space is itself weakly compact. See Communications de l'Institut des Sciences Mathématiques et Méchaniques de l'Université de Kharkoff et la Société Mathématique de Kharkoff, (4), vol. 14 (1937), pp. 239-242.

$\left({ }^{10}\right)$ One can readily obtain this result by employing an argument similar to that used by Dunford [8, p. 644]. 
5.6. Corollary. If $U$ is weakly completely continuous on $L$ to $L$, then $U^{2}$ is completely continuous.

$U$ takes the unit sphere in $L$ into a conditionally weakly compact subset of $L$, and by Theorem 5.5 its iterate takes this subset into a conditionally compact subset of $L$. In other words $U^{2}$ is completely continuous.

A uniform mean erogodic theorem for weakly completely continuous transformations on $L$ to $L$ is easily obtainable by means of Corollary 5.6 and a mean ergodic theorem due to Kakutani [16] and Yosida [28].

6. On completely continuous transformations. In this section we make further application of our study, demonstrating that each completely continuous transformation on any of the spaces $L^{p}, l^{p}(1 \leqq p \leqq \infty), C, c_{0}, M_{T}$ to an arbitrary Banach space $X$ can be approximated in the norm by degenerate transformations (see footnote on p. 526). The notation is that of $\S 5$.

For $\dot{x} \varepsilon V^{q}(X)(1<q<\infty)$ we define

$$
x_{\pi}(\tau)=\sum_{\pi} \frac{x\left(\tau_{i}\right)}{\left|\tau_{i}\right|} \cdot\left|\tau \cdot \tau_{i}\right|
$$

and for $\dot{x} \varepsilon V^{\infty}(X)$ we define

$$
x_{\pi}(\tau)=\sum_{\pi} \frac{x\left(\sigma_{i}\right)}{\left|\sigma_{i}\right|} \cdot\left|\tau \cdot \tau_{i}\right| \quad\left(\sigma_{i}<\tau_{i},\left|\sigma_{i}\right|<\infty\right) .
$$

Clearly $\dot{x}_{\pi} \varepsilon V_{c}^{q}(X)$.

6.1. Theorem. If $\dot{x} \varepsilon V_{c}^{q}(X)(1<q \leqq \infty)$, then $\lim _{\pi}\left\|\dot{x}_{\pi}-\dot{x}\right\|=0$.

By definition, the set $[\bar{x}(x(\tau)) \mid\|\bar{x}\| \leqq 1]$ where $\dot{x} \varepsilon V^{q}{ }_{c}(X)$ is a conditionally compact subset of $V^{q}(R)$. If we use the usual isometric correspondence between $V^{q}(R)$ and $L^{q}$, it follows immediately from Theorem 3.7 that

$$
\lim _{\pi}\left\|\dot{x}_{\pi}-\dot{x}\right\|=\lim _{\pi} \text { L.U.B. }\left[\left\|\bar{x}\left(\dot{x}_{\pi}\right)-\bar{x}(\dot{x})\right\| \mid\|\bar{x}\| \leqq 1\right]=0 .
$$

6.2. CoROllaRY. If $U$ is a completely continuous transformation on $L^{p}$ to $X$ $(1 \leqq p<\infty)$, then

(1) $U(\phi)=\int \phi d x$ where $\dot{x} \varepsilon V_{c}^{q}(X)$

(2) If $U_{\pi}(\phi)=\sum_{\pi} \int_{\tau_{i}} \phi d \alpha \cdot x\left(\tau_{i}\right) /\left|\tau_{i}\right|(1<p<\infty)$ or if $U_{\pi}(\phi)=\sum_{\pi} \int_{\tau_{i}} \phi d \alpha$ $\cdot x\left(\sigma_{i}\right) /\left|\sigma_{i}\right|(p=1)$, then $\lim _{\pi}\left\|U_{\pi}-U\right\|=0$.

This is a consequence of Theorems 4.3 and 6.1.

For notational convenience, we write $c_{0}=l^{\infty}$.

6.3. THEOREM. If $U$ is a completely continuous transformation on $l^{p}$ $(1 \leqq p \leqq \infty)$ to $X$, then

(1) $U(a)=\sum_{i=1}^{\infty} a_{i} x_{i}$ where $\dot{x} \varepsilon v^{q}{ }_{c}(X)$,

(2) if $U_{n}(a)=\sum_{i=1}^{n} a_{i} x_{i}$, then $\lim _{n}\left\|U_{n}-U\right\|=0$. 
For $p=\infty$, this is a consequence of Theorems 1.7 and 4.7. For $1 \leqq p<\infty$, the theorem is a special case of Corollary 6.2.

6.4. Theorem. If $\dot{x} \varepsilon V_{c}^{1}(X)$, then there exists a non-negative $\beta(\tau) \varepsilon V^{1}(R)$ such that

$$
\lim _{\pi} \sum_{\pi} \frac{x\left(\tau_{i}\right)}{\beta\left(\tau_{i}\right)} \beta\left(\tau \cdot \tau_{i}\right)=\dot{x}
$$

where $\pi$ is of type 2.

By the definition of $V^{1}{ }_{c}(X), S \equiv[\bar{x}(x(\tau)) \mid\|\bar{x}\| \leqq 1]$ is a conditionally compact subset of $V^{1}(R)$. Hence there exists the sequence $\left[\bar{x}_{n} \mid\left\|\bar{x}_{n}\right\| \leqq 1\right.$, $n=1,2, \cdots]$ such that $\bar{x}_{n}(x(\tau))$ is dense in $S$. Let $\beta_{n}(\tau)$ be the absolute variation of $\bar{x}_{n}(x(\tau))$. Define $\beta(\tau)=\sum_{1}^{\infty}\left(1 / 2^{n}\right) \beta_{n}(\tau)$. Clearly $\beta(\tau) \varepsilon V^{1}(R)$ and $\left|\beta_{n}(\tau)\right| \leqq 2^{n}|\beta(\tau)| . \bar{x}_{n}(x(\tau))$ is therefore absolutely continuous with respect to $\beta(\tau)$. The class of all elements of $V^{1}(R)$ absolutely continuous with respect to $\beta(\tau)$ form a closed linear space $A C(\beta)$. Let $\pi=\left(\tau_{1}, \tau_{2}, \cdots, \tau_{n}\right)$ be of type 2 and such that $\beta\left(\tau_{i}\right) \neq 0(i=1,2, \cdots, n)$. Define

$$
U_{\pi}(\gamma(\tau))=\sum_{\pi} \frac{\gamma\left(\tau_{i}\right)}{\beta\left(\tau_{i}\right)} \beta\left(\tau \cdot \tau_{i}\right)
$$

on $A C(\beta)$ to $A C(\beta)$. Then $\left\|U_{\pi}\right\| \leqq 1$. By a theorem due to Bochner [4, pp. 780-783] $\lim _{\pi} U_{\pi}(\gamma(\tau))=\gamma(\tau)$ for all $\gamma(\tau) \varepsilon A C(\beta)$. By Theorem 3.7, $\lim _{\pi} U_{\pi}\left(\bar{x}_{n}(x(\tau))\right)=\bar{x}_{n}(x(\tau))$ uniformly in $n$ and hence

$$
\lim _{\pi}\left\|\sum_{\pi} \frac{x\left(\tau_{i}\right)}{\beta\left(\tau_{i}\right)} \beta\left(\tau \cdot \tau_{i}\right)-\dot{x}\right\|=\lim _{\pi} \underset{n}{\text { L.U.B. }}\left[\left\|U_{\pi}\left[\bar{x}_{n}(x(\tau))\right]-\bar{x}_{n}(x(\tau))\right\|\right]=0 \text {. }
$$

6.5. CoROllaRY. If $U$ is a completely continuous transformation on $M_{T}$ [or $\left.L^{\infty}\right]$ to $X$, then

(1) $U(\phi)=\int \phi d x$ where $\dot{x} \varepsilon V^{1}{ }_{c}(X)$,

(2) there exists $a \beta(\tau) \varepsilon V^{1}(R)$ such that if

$$
U_{\pi}(\phi)=\sum_{\pi} \frac{\int_{\tau_{i}} \phi d \beta}{\beta\left(\tau_{i}\right)} x\left(\tau_{i}\right),
$$

then $\lim _{\pi}\left\|U_{\pi}-U\right\|=0$.

This is a consequence of Theorems 4.3 and 6.4.

6.6. COROLlaRY. If $U$ is a completely continuous transformation on $C$ to $X$, then $U$ is approximable in the norm by degenerate transformations.

According to a result of Gelfand's [12, p. 283], $U(\phi)=\int \phi d x$ where $\dot{x} \varepsilon V_{c}(X)$ and $\|\dot{x}\|=\|U\|$ (see end of $\S 2$ ). Now $\lim _{t \rightarrow t^{+}} \bar{x}(x(t))$ and $\lim _{t \rightarrow t^{-}} \bar{x}(x(t)$ ) exist by virtue of $\bar{x}(x(t))$ 's being of bounded variation. Since the values as- 
sumed by $x(t)$ form a conditionally compact set, it follows that $x\left(t^{+}\right)$and $x\left(t^{-}\right)$ are defined for all $t$. Let $\tau$ be the Jordan field of sets $\tau$ generated by all open intervals and points of $(0,1)$. If $\tau$ consists of the disjoint sets $\left[\left(a_{1}^{+}, b_{1}^{-}\right), \cdots\right.$, $\left.\left(a_{n}^{+}, b_{n}^{-}\right) ; c_{1}, \cdots, c_{m}\right]$, define

$$
x(\tau)=\sum_{i=1}^{n}\left[x\left(\overline{b_{i}}\right)-x\left(a_{i}^{+}\right)\right]+\sum_{i=1}^{m}\left[x\left(c_{i}^{+}\right)-x\left(\overline{c_{i}}\right)\right] .
$$

Clearly $x(\tau) \varepsilon V_{c}^{1}(X), U(\phi)=\lim _{\pi} \sum_{\pi} \phi\left(t_{i}\right) x\left(\tau_{i}\right)$ ( $\pi$ of type 2$)$, and $\|U\|=\|\dot{x}\|$. The remainder of the argument follows from Theorem 6.4.

We remark that in Theorems 6.1, 6.4 and Corollaries 6.2, 6.5, 6.6 the $\pi$-limit can be replaced by a sequential limit.

The problem of approximating completely continuous transformations on $X$ to certain spaces $Y$ by degenerate transformations has been investigated by Maddaus [19]. He shows that this is possible whenever there exist degenerate transformations $V_{n}$ such that $\lim _{n \rightarrow \infty} V_{n}(y)=y$ for all $y \varepsilon Y$

Let $Y$ be a Banach space possessing a generalized base, $U_{\pi}$ (see $\S 3$ ). Suppose $U$ is a completely continuous transformation on $X$ to $Y$. Then the set $S \equiv[U(x) \mid x \varepsilon X,\|x\| \leqq 1]$ is conditionally compact. By Theorem 3.7 $\lim _{\pi} U_{\pi}(U(x))=U(x)$ uniformly for all $x \varepsilon X$ for which $\|x\| \leqq 1$. It follows that $\lim _{\pi}\left\|U_{\pi}(U)-U\right\|=0$. As $U_{\pi}(U)$ is a degenerate transformation, this gives Maddaus's result in a slightly more general form.

7. On the extension of linear transformations. If $U$ is a linear transformation on $X$ to $Y$ and $Z$ contains $X$ as a proper subspace, then a linear transformation $U_{1}$ on $Z$ to $Y$ such that $U(z)=U_{1}(z)$ for all $z \varepsilon X$ is called an extension of $U$. Any Banach space $Y$ can be imbedded( $\left.{ }^{11}\right)$ in a space of type $M_{T}\left({ }^{12}\right)$. We will designate such a space which contains $Y$ or its image under an equivalence by $M_{T} \supset Y$.

7.1. TheOREM. The general form of the linear transformation $U$ on $X$ to $M_{T}$ is

$$
U(x)=\left[\bar{x}_{t}(x)\right]
$$

where $\|U\|=$ L.U.B. $\left[\left\|\bar{x}_{t}\right\| \mid t \varepsilon T\right]$.

For every $t \varepsilon T$ there exists a linear functional $\bar{\alpha}_{t}$ such that $\bar{\alpha}_{t}(a)=a(t)$. Let $\bar{x}_{t}=\bar{U}\left(\bar{\alpha}_{t}\right)$. Then $a(t)=\bar{\alpha}_{t}[U(x)]=\bar{x}_{t}(x)$ and $\|U\|=$ L.U.B. $\left[\left|\bar{\alpha}_{t}[U(x)]\right|=\left|\bar{x}_{t}(x)\right| \mid t \varepsilon T,\|x\| \leqq 1\right]=$ L.U.B. $\left[\left\|\bar{x}_{t}\right\| \mid t \varepsilon T\right]$.

7.2. Corollary. Any linear transformation $U$ on $X$ to $M_{T}$ has an extension $U_{1}$ on $Z \supset X$ to $M_{T}$ such that $\|U\|=\left\|U_{1}\right\|$.

(11) By an imbedding of $Y$ into a subspace $Z$ of $M$ we shall mean that $Y$ is equivalent [1, p. 180$]$ to $Z$.

(12) Let $T \equiv \Gamma_{1}$, the unit sphere of some determining manifold in $\bar{Y}$. Then $U(y)=\bar{y}(y)$ on $Y$ to $M_{T}$ defines an equivalence between $Y$ and a subset of $M_{T}$. 
By the Hahn-Banach theorem [1, p. 55, Theorem 2], $\bar{x}_{t}$ has a norm preserving extension $\bar{z}_{t}$ on $Z . U_{1}(z)=\left[\bar{z}_{t}(z)\right]$ is the required extension.

7.3. CoRollary. If $Y$ is isomorphic with $M_{T}$, then any linear transformation $U$ on $X$ to $Y$ has an extension on $Z$ to $Y$.

As $Y$ and $M_{T}$ are isomorphic $[1$, p. 180] there exists a biunique and bicontinuous linear transformation $V$ on $Y$ into the entire space $M_{T}$. $V U$ is then a linear transformation on $X$ to $M_{T}$ which by Corollary 7.2 has the extension $(V U)_{1}$ on $Z$ to $M_{T}$. It is clear that $V^{-1}(V U)_{1}$ is the required extension on $Z$ to $Y$.

7.4. Corollary. Any linear transformation $U$ on $X$ to $Y$ has an extension on $Z \supset X$ to $Y$ if either of the following is true:

(1) There exists a projection transformation $\left({ }^{13}\right) P$ on $Z$ to $X$.

(2) There exists a projection transformation $P$ on $M_{T} \supset Y$ to $Y$.

If (1) holds then $U_{1}=U P$ is the required extension on $Z \overline{\text { to } Y \text {. If (2) holds }}$ and $U_{1}$ is the extension of Corollary 7.2 on $Z$ to $M_{T} \supset Y$, then $P U_{1}$ is the required extension on $Z$ to $Y$.

In view of Corollary 7.4, the existence of projection transformations on spaces $M_{T} \supset Y$ to $Y$ assumes importance in the study of the extension of linear transformations. As yet we can give only negative results in this direction.

Fichtenholtz and Kantorovitch [10, p. 92] have proved that there does not exist a projection transformation on $M_{T}$ to $C$ where $T=(0,1)$ and $C$ is the space of continuous functions on $(0,1)$. Banach and Mazur $[2, p .111]$ have shown that for a separable space $Y$ whose conjugate space is not weakly complete there does not exist a projection transformation on $C$ to any imbedding of $Y$ in $C$. Consequently there exists no projection transformaton on $M_{T} \supset Y$ to an imbedding of $Y$ which is contained in an imbedding of $C$ in $M_{T} \supset Y$.

If there existed a projection transformation on the space $C_{1}$ of functions on $(0,1)$ having only discontinuities of the first kind to $C$ then the methods of Gelfand $[12$, p. 281] would show that the identity transformation on $C$ to $C$ could be expressed in the form $U(\phi)=\int \phi d x$ where $\dot{x} \varepsilon V(X)$. The example at the end of $\S 4$ shows that this is not the case. Therefore there exists no projection transformation on $M_{T} \supset C$ to an imbedding of $C$ which is contained in an imbedding of $C_{1}$ in $M_{T} \supset C$.

\subsection{There exists no projection transformation on $m$ to $c$.}

If there existed a projection transformation $P$ on $m$ to $c$, then any weakly convergent sequence of linear functionals $\left\{\bar{a}_{p}\right\}$ on $c$ corresponds to a se-

(13) A projection transformation $P$ is a linear transformation with the property that $P^{2}=P$. 
quence of extensions $\left\{\bar{x}_{p}=\bar{P}\left(\bar{a}_{p}\right)\right\}$ which is weakly convergent on $m$. Now $\bar{a}_{p}(a)=a(p+1)-a(p)$ converges weakly to zero on $c$. Using the notation of Corollary 3.4, we have $\bar{x}_{p}(x)=\int_{T} x(t) d \beta^{p}$ and $\sum_{t}\left|\beta^{p}(t)\right| \rightarrow 0$. Since $x_{p}(a)=a_{p}(a)$ $=a(p+1)-a(p)$, it follows that $\beta^{p}(p+1)=1=-\beta^{p}(p)$ which is contrary to the above. There can therefore exist no projection transformation on $m$ to $c$.

\section{REFERENCES}

1. S. Banach, Théorie des Opérations Linéaires, Warsaw, 1932.

2. S. Banach and S. Mazur, Zur Theorie der linearen Dimension, Studia Mathematica, vol. 4 (1933), pp. 100-112.

3. Garrett Birkhoff, Integration in a Banach space, these Transactions, vol. 38 (1935), pp. $357-378$

4. S. Bochner, Additive set functions on groups, Annals of Mathematics, (2), vol. 40 (1939), pp. 769-799.

5. S. Bochner and A. E. Taylor, Linear functionals on certain spaces of abstractly-valued functions, Annals of Mathematics, (2), vol. 39 (1938), pp. 913-944.

6. N. Dunford, Integration and linear operations, these Transactions, vol. 40 (1936), pp. $474-494$

7. - - Uniformity in linear spaces, these Transactions, vol. 44 (1938), pp. 305-356.

8. - A mean ergodic theorem, Duke Mathematical Journal, vol. 5 (1939), pp. 635-646.

9. N. Dunford and B. J. Pettis, Linear operations among summable functions, Proceedings of the National Academy of Sciences, vol. 25 (1939), pp. 544-550.

10. G. Fichtenholtz and L. Kantorovitch, Sur les opérations linéaires dans l'espace des functions bornées, Studia Mathematica, vol. 5 (1934), pp. 69-98.

11. M. Fréchet, Les ensembles abstraits et le calcul fonctionnel, Rendiconti del Circolo Matematico di Palermo, vol. 30 (1910), p. 19.

12. I. Gelfand, Abstrakte Funktionen und lineare Operatoren, Recueil Mathématique, vol. 4 (1938), pp. 235-284.

13. M. Gowurin, Stieltjessche integration, Fundamenta Mathematicae, vol. 27 (1936), pp. 254-268.

14. T. H. Hildebrandt, On bounded linear functional operations, these Transactions, vol. 36 (1934), pp. 868-875.

15. T. H. Hildebrandt and I. J. Schoenberg, On linear functional operations and the moment problem for a finite interval in one or several dimensions, Annals of Mathematics, (2), vol. 34 (1933), pp. 317-328.

16. Shizuo Kakutani, Iteration of linear operations in complex Banach spaces, Proceedings of the Imperial Academy of Tokyo, vol. 14 (1938), pp. 295-300.

17. L. Kantorovitch and B. Vulich, Sur la représentation des opérations linéaires, Composito Mathematica, vol. 5 (1937), pp. 119-165.

18. A. Kolmogoroff, Über Kompaktheit der Funktionenmengen bei der Konvergenz im Mittel, Nachrichten der Gesellschaft der Wissenschaften zu Göttingen, 1931, pp. 60-63.

19. I. Maddaus, On completely continuous linear transformations, Bulletin of the American Mathematical Society, vol. 44 (1938), pp. 279-282.

20. E. H. Moore and H. L. Smith, $A$ general theory of limits, American Journal of Mathematics, vol. 44 (1922), pp. 102-121.

21. W. Orlicz, Beiträge zur Theorie der Orthogonalentwicklungen II, Studia Mathematica, vol. 1 (1929), pp. 241-255. 304.

22. B. J. Pettis, On integration in vector spaces, these Transactions, vol. 44 (1938), pp. 277- 
23. R. S. Phillips, On integration in a linear convex topological space, these Transactions, vol. 47 (1940), pp. 114-146.

24. J. Radon, Theorie und Anwendung der absolut additiven Mengenfunktionen, Sitzungsberichte der Akademie der Wissenschaften, Vienna, Class IIa, vol. 122 (1913), p. 1384.

25. M. Riesz, Sur les ensembles compacts de fonctions sommables, Acta Szeged, vol. 6 (1933), pp. 136-142.

26. J. Tamarkin, On compactness of the space $L^{p}$, Bulletin of the American Mathematical Society, vol. 38 (1932), pp. 79-84.

27. A. Tulajkov, Zur Kompaktheit im Raum $L^{p}$ für $p=1$, Nachrichten der Gesellschaft der Wissenschaften zu Göttingen, 1933, pp. 167-170.

28. Kôsaku Yosida, Mean ergodic theorem in Banach spaces, Proceedings of the Imperial Academy of Tokyo, vol. 14 (1938), pp. 292-294.

The Institute for Advanced Study, Princeton, N. J. 